\title{
Etude des phénomènes d'entraînement d'air. Application aux évacuateurs de crue
}

\author{
Hubert Chanson
}

\section{Introduction}

\subsection{Présentation}

Les barrages sont protégés des crues par un système de décharge appelé évacuateur de crues. Généralement il s'agit d'un évacuateur de crues dit de surface. Un évacuateur de crues de surface est un déversoir situé en crête de barrage. Il comporte: 1) une entrée, 2) un coursier et 3) un système de dissipation d'énergie. L'écoulement obtenu sur la rampe est un écoulement à surface libre supercritique (grand nombre de Froude: $F r \gg 1)$.

Avec l'accroissement de la hauteur des barrages, les vitesses d'écoulement sur le coursier peuvent atteindre $50 \mathrm{~m} / \mathrm{s}$. Dans ces conditions, les dommages dus à l'érosion par cavitation sont inacceptables. Des études ont montrées que les dangers d'érosion par cavitation apparaissent pour des vitesses supérieures à $15 \mathrm{~m} / \mathrm{s}$.

Pour prévenir ou ralentir cette érosion, il est possible d'utiliser des bétons spéciaux (à haute résistance), des revêtements d'acier, des surfaçages en résine polymère ou epoxy. Ces solutions sont coûteuses et ne sont pas satisfaisantes pour des vitesses supérieures à $30 \mathrm{~m} / \mathrm{s}$. Ce type de protection est en outre dépendant des conditions de vieillissement des revêtements de surface.

Une autre méthode consiste à augmenter la compressibilité du fluide près de la surface du coursier en introduisant de l'air dans l'écoulement. Cette disposition est parfois moins coûteuse si elle est prévue dès le début du projet et n'est pas soumise aux contraintes de vieillissement de la surface de l'évacuateur de crues.

Dans une première partie, les phénomènes d'entrainement d'air au-dessus d’un aérateur seront étudiés, à l'aide de résultats expérimentaux obtenus sur le modèle de l'évacuateur de crues du barrage de Clyde (NouvelleZélande). Puis les mesures de STRAub et ANDERSON (1958), et CAIN (1978), pour des écoulements aérés à surface libre, seront analysées en suivant la méthode développée par Wo(1) (1985), incluant des corrections et extensions.

A study of air entrainment. Application to spilway design.

Cavitation erosion damage to spillway surfaces may be prevented with the use of aeration devices, called aerators. These serve to introduce air in the layers close to the channel bottom in order to reduce the cavitation damage.

The performances of spillway aerator are studied with data obtained on a steep spillway model and high velocities (from $4 \mathrm{~m} / \mathrm{s}$ up to $15 \mathrm{~m} / \mathrm{s}$ ). Air concentration and velocity profiles at various cross-sections above and downstream of the aerator are presented and analyzed. They provide a better understanding of the air entrainment processes occurring above a bottom aerator.

A new analysis of the flow downstream of an aerator is presented and this contributes to a new method for the determination of aerator spacing. 


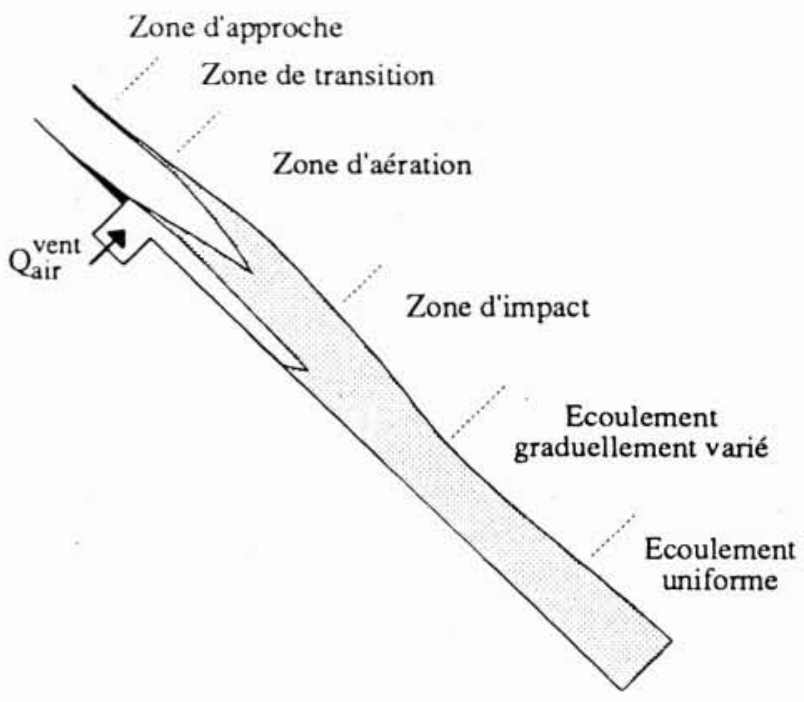

1. Evacuateur de crues avec un aérateur de fond.

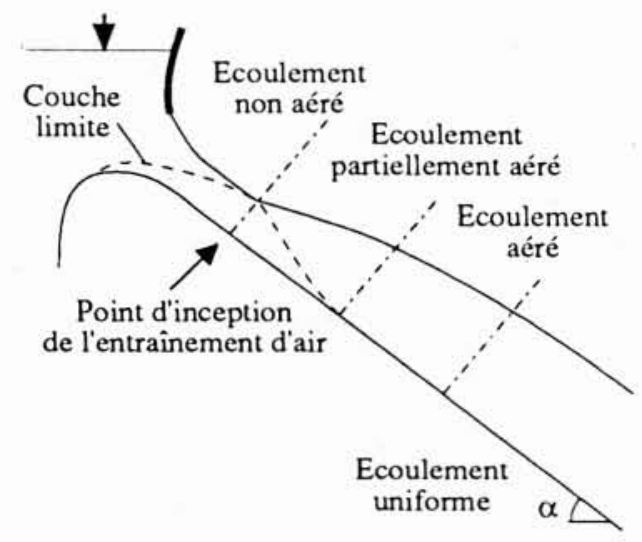

2. Evacuateur de crues avec aération naturelle.

Ensuite une étude de l'écoulement graduellement varié en aval d'un aérateur (fig. 1) sera développée suivant l'analyse de WOOD (1985) pour un écoulement graduellement varié en avant d'un point d'inception (fig. 2). Enfin l'application pratique de ces résultats sera discutée dans le cadre de la protection du coursier d'un évacuateur de crues contre l'érosion par cavitation.

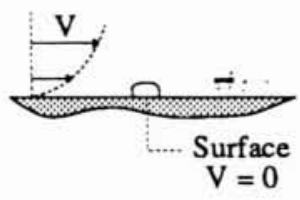

3. Contraintes de cisaillement sur une bulle d'air à la surface du coursier.

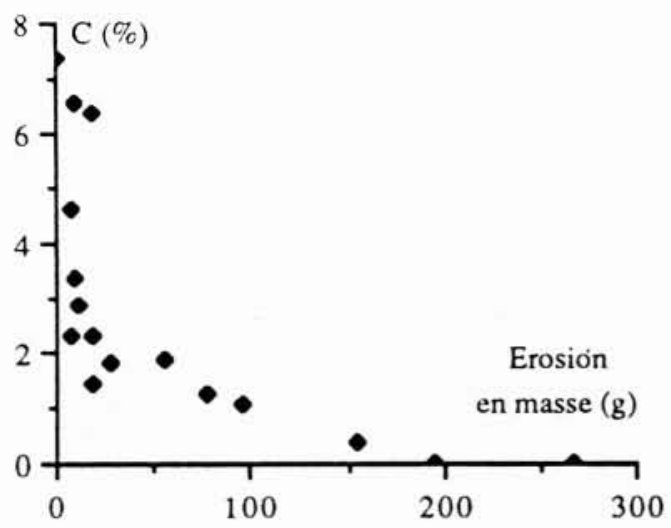

4. Relation entre l'érosion par cavitation et la concentration en air pour $V=30,5 \mathrm{~m} / \mathrm{s}$. PETERKA (1953).

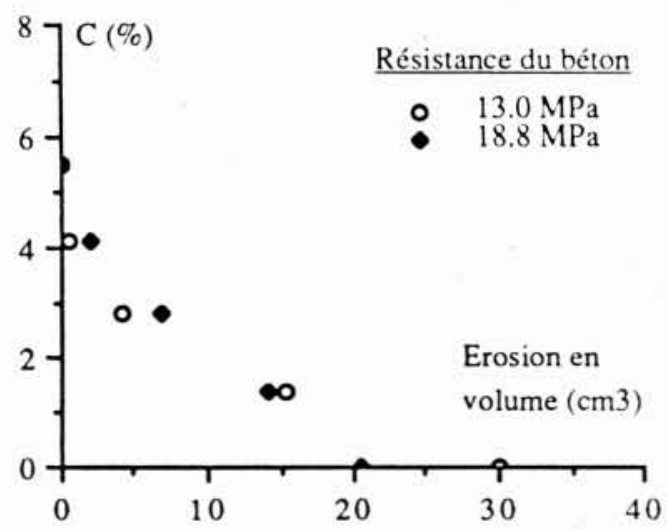

5. Relation entre l'érosion par cavitation et la concentration en air pour $V=46 \mathrm{~m} / \mathrm{s}$. RUSSELL et SHEEHAN (1974). 


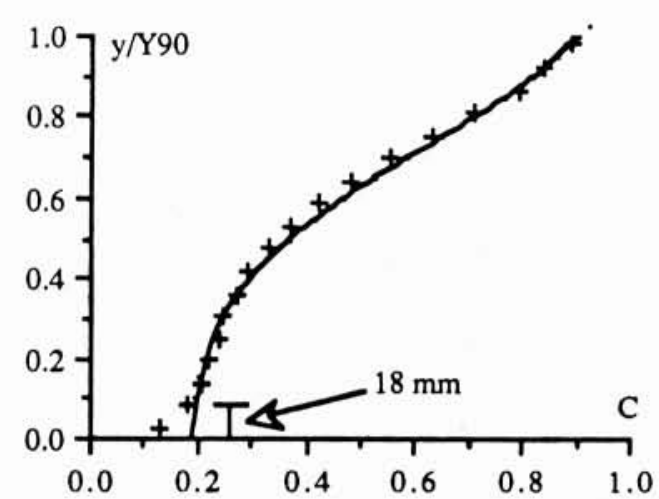

a. Barrage d'Aviemore. CAIN (1978).

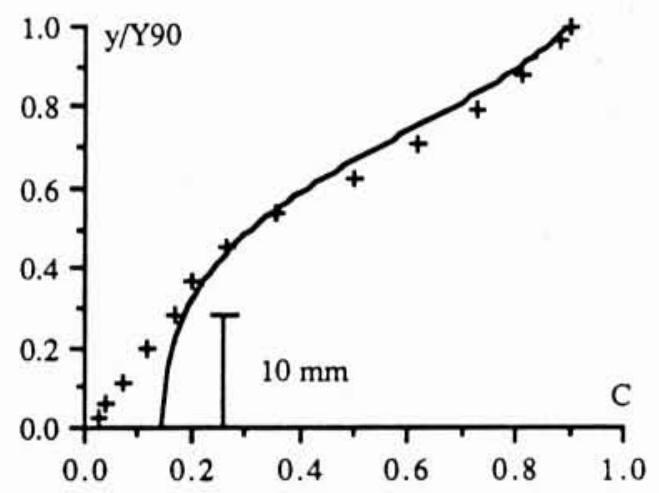

b. Modèle 1/15ème. CHANSON (1988b).

6. Distributions de concentration en air près de la surface d'un évacuateur de crues.

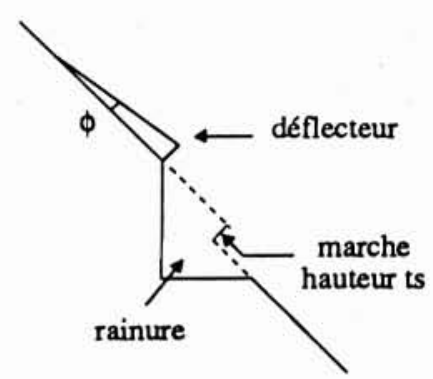

7. Schéma d'un aérateur de fond.

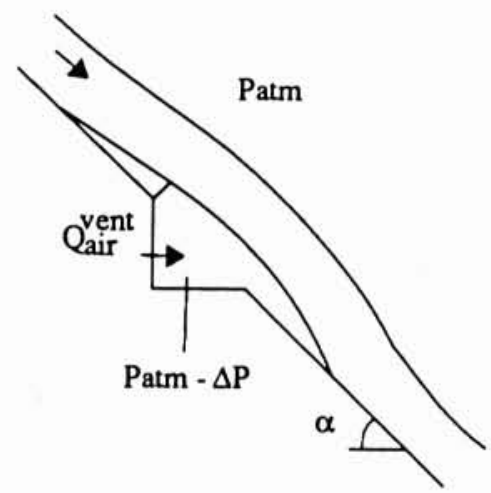

8. Ecoulement au-dessus d'un aérateur.
Au contact du coursier, la concentration en air est nulle. En effet les contraintes de cisaillement appliquée à une bulle d'air à la surface du coursier, la désagrégeraient en minuscules bulles de volume négligeable (fig. 3). Des mesures de concentration en air obtenues sur prototype (CAIN 1978) et sur modèle (CHANSON 1988b) sont portées sur les figures 6 et comparées avec le profil théorique d'écoulement uniforme (détaillé plus loin). Les résultats suggèrent la présence d'une couche limite de concentration en air de 10 à $15 \mathrm{~mm}$ d'épaisseur près de la surface du coursier.

Pour des conditions d'expérimentation données, les figures 4 et 5 indiquent que l'érosion par cavitation est fortement réduite si la concentration en air près de la surface du coursier est supérieure à 1-2\%, et l'érosion est stoppée pour des concentrations en air supérieure à 6-8\%. En présence d'une épaisse couche limite de concentration en air, des concentrations en air plus importantes peuvent être nécessaires hors de cette couche limite.

Si l'entraînement naturel d'air à la surface libre de l'écoulement est suffisamment important, de telles conditions peuvent être atteintes. Toutefois, quand cette aération de surface est insuffisante, ou si les vitesses sont trop élevées (ex. : $V>30 \mathrm{~m} / \mathrm{s}$ ), une quantité additionnelle d'air doit être introduite artificiellement. Ceci est réalisé à l'aide de dispositifs de ventilation par le fond ou par les côtés, appelés aérateurs.

\subsubsection{Dispositifs de ventilation-aérateurs}

Un aérateur de fond comprend généralement (fig. 7) : 1) un déflecteur (angle $\phi)$, 2) une marche (hauteur $t_{s}$ ) et 3) une rainure. Un déflecteur est bien adapté aux petits et moyens débits. Une marche est plus efficace pour les grands débits. La rainure améliore les conditions d'alimentation en air de l'aérateur.

A la fin du déflecteur l'écoulement devient un jet libre turbulent. Une cavité, soumise à une dépression naturelle $(\Delta P)$, se développe sous la surface libre inférieure du jet (fig. 8) et une ventilation naturelle (ou forcée) s'établit dans la cavité ( $\left.Q_{\text {air }}^{\text {vent }}\right)$.

VOLKART et CHERVET (1983) ont étudié sur modèle un grand nombre de configurations d'aérateur, et VOLKART et RUTSCHMANN (1984) présentent plusieurs exemples de dispositifs de ventilation de la cavité. 


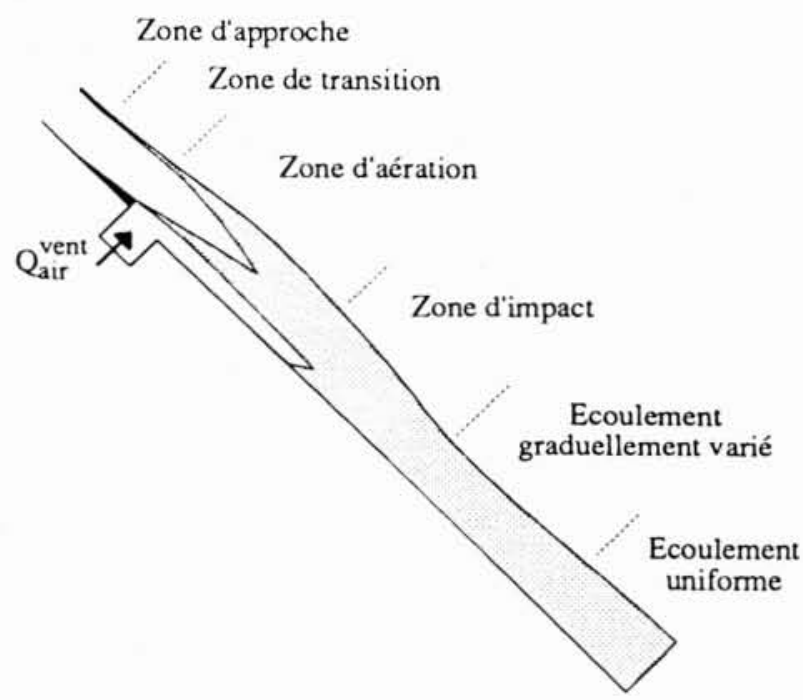

9. Principales régions d'écoulement.

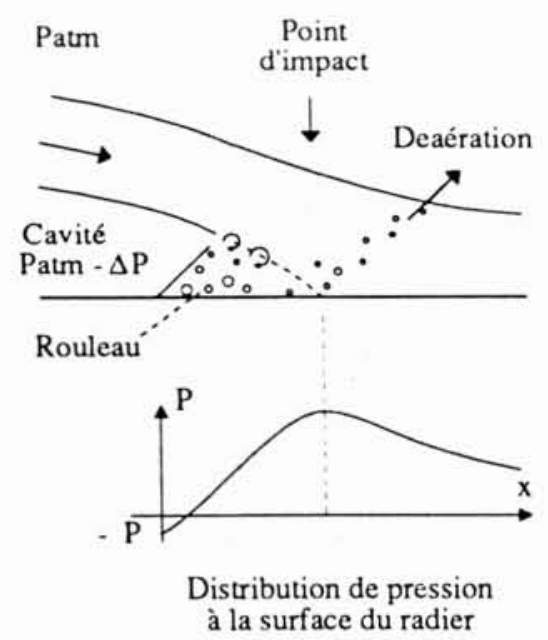

11. Point d'impact du jet libre.
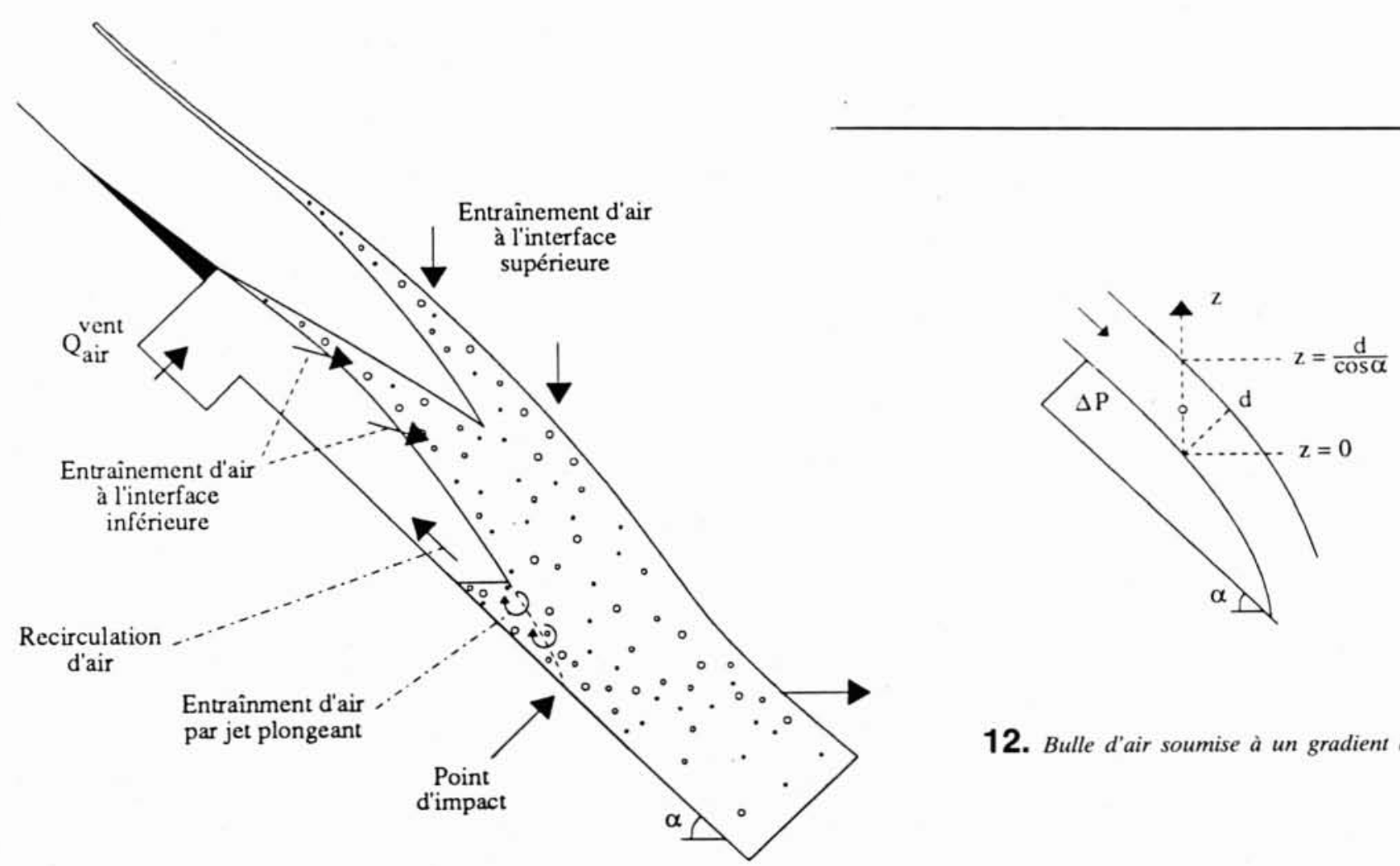

10. Principaux mécanismes d'entrainement d'air. 


\subsection{Définition}

La concentration en air $C$ est définie comme le volume d'air par unité de volume. On définit la profondeur de référence $d$ comme :

$$
d=\int_{0}^{Y_{(0)}}(1-C) \mathrm{d} y
$$

où y est mesuré perpendiculairement à la surface de l'évacuateur de crues, et $Y_{90}$ est une autre profondeur de référence définie pour $C=90 \%$. La concentration en air moyenne $C_{m}$ dans une section transversale à l'écoulement est définie par:

$$
\left(1-C_{m}\right) Y_{90}=d .
$$

La vitesse moyenne $V_{m}$ de l'écoulement dans une section est déduite de :

$$
V_{m}=\frac{q_{\mathrm{cau}}}{d}
$$

Le débit en eau par unité de largeur $q_{\text {cau }}$ devient :

$$
q_{\mathrm{cau}}=\left(1-C_{m}\right) V_{m} Y_{q_{0}} .
$$

Enfin une autre vitesse de référence $\left(V_{90}\right)$ est définie comme celle à $Y_{90}$.

\section{Les phénomènes d'entraînement d'air}

\subsection{Description}

L'écoulement au-dessus d'un aérateur comporte plusieurs régions (fig. 9): 1) la zone d'approche, 2) la zone de transition, 3) la zone d'aération, 4) la zone d'impact, 5) la région d'écoulement graduellement varié et 6) la région d'écoulement uniforme loin en aval.

La région d'approche caractérise les conditions initiales de l'écoulement au-dessus de l'aérateur et la surface de l'écoulement peut être partiellement aérée. La zone de transition coïncide avec la longueur du déflecteur. Le déflecteur affecte le champ de pression et de turbulence dans une section perpendiculaire à la surface de l'évacuateur de crues. Ceci modifient principalement les conditions à la surface libre inférieure du jet libre.

Avec ou sans déflecteur, l'écoulement, à la naissance du jet libre, est sujet à une rapide redistribution du champ de pression, d'un profil quasi hydrostatique à un gradient négatif de pression (fig. 8).

Dans la zone d'aération l'air est entrainé principalement par diffusion turbulente au long des interfaces aireau inférieure et supérieure ( $f i g$. 10). A la naissance du jet libre, on observe une partie centrale non aérée qui s'amenuise au fur et à mesure que l'écoulement est aéré le long des surfaces libres. Cette région, appelée la zone d'aération des surfaces libres, est caractérisée par l'impossibilité de transfert d'air entre les régions supérieure et inférieure du jet libre.

A la fin du jet libre, une quantité additionnelle d'air est entrainée par jet plongeant à l'intersection du jet avec les rouleaux (fig. 10). La zone d'impact du jet est caractérisée par une redistribution rapide des profils de concentration en air et de vitesse, ainsi que par une redistribution rapide de pression à la surface de l'évacuateur de crues (fig. 11), d'un gradient négatif de pression dans la cavité ventilée à un maximum de pression au point d'impact, et finalement un profil hydrostatique loin en aval.

En aval, dans la région d'écoulement graduellement varié, les profils de concentration en air et de vitesse se redistribuent lentement et tendent vers des profils d'équilibre dans la région d'écoulement uniforme.

\subsection{Les mécanismes d'entraînement d'air}

Dans la zone d'aération, les principaux mécanismes d'entrainement d'air sont (fig. 10) : 1) l'entrainement d'air par diffusion turbulente au long des interfaces inférieure et supérieure du jet libre, 2) l'entraînement d'air par jet plongeant (ou par effet de coin) quand le jet réattache la surface de l'évacuateur de crues, et 3) une recirculation d’air dans la cavité ventilée.

Entrainement d'air à l'interface supérieure: Quand le jet libre quitte le déflecteur, l'écoulement, initialement soumis à un gradient de pression quasi hydrostatique, devient sujet à un gradient de pression négatif ( fig. 12) :

$$
\frac{\mathrm{d} P}{\mathrm{~d} z}=\frac{\Delta P}{d} \cos \alpha .
$$

Le mouvement d'une bulle d'air, soumise à ce gradient de pression, devient une chute libre, et, pour des bulles sphériques soumises à un gradient de pression linéaire (equation 5), la vitesse limite d'une bulle de gaz $u_{r}$ est égale à (CHANSON 1988) :

$u_{r}^{2}=\frac{4 g d_{b}}{3 C_{d}}$ Signe $\left\{\mathrm{P}_{\mathrm{N}} \cos \alpha+\frac{\rho_{\text {air }}}{\rho_{\text {cau }}}\right\} \times\left|P_{N} \cos \alpha+\frac{\rho_{\text {air }}}{\rho_{\text {cau }}}\right|$

où $C_{d}$ est le coefficient de trainée d'une bulle d'air, $d_{b}$ est le diamètre d'une bulle d'air, et $P_{N}$ est le gradient adimensionel de pression : $P_{N}=\frac{\Delta P}{\rho_{\text {cau }} g d}$.

Entrainement d'air à l'interface inférieure: A l'interface inférieure du jet libre, la réduction brutale des contraintes de cisaillement est un phénomène dominant. Les interactions entre les effets du rapide changement de gradient de pression et de la recirculation d'air dans la cavité sont des paramètres importants encore mal définis.

Entrainement d'air par jet plongeant: Quand le jet libre ré-attache la surface de l'évacuateur de crues, des poches et bulles d'air sont emprisonnées à l'intersection du jet libre avec les rouleaux, et sont entraînées en aval. Des études précédentes (CASTELEYN et al. 1977, ...) ont montrées que la quantité d'air entraînée par un jet libre heurtant une surface libre, s'exprime sous la forme $Q_{\text {air }}=K\left(V-V_{c}\right)^{n}$, où $V$ est la vitesse du jet libre, $K$ une constante et $V_{c}$ la vitesse critique à laquelle l'entraînement d'air commence $\left(V_{c} \sim 0.8 \mathrm{~m} / \mathrm{s}\right)$. 


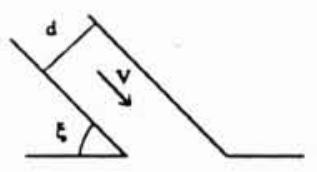

13. Entrainement d'air par jet plongeant.

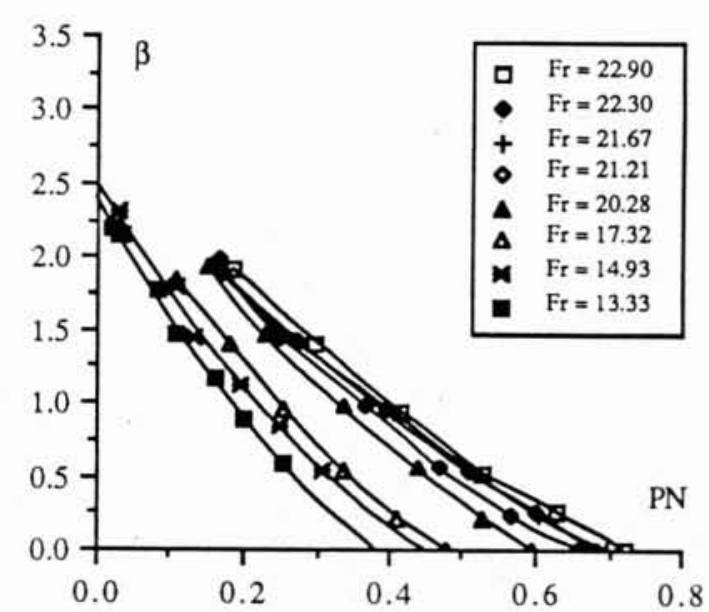

15. Demande en air pour $d / t_{s}=0,95$. Aérateur avec un déflecteur $\left(\phi=5,7^{\circ}\right)$. CHANSON (1988).

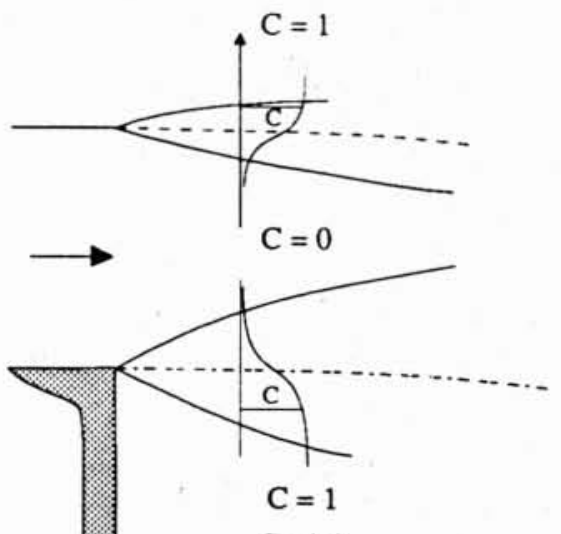

Cavité

17. Distributions de concentration en air dans la zone d'entrânement des surfaces libres.

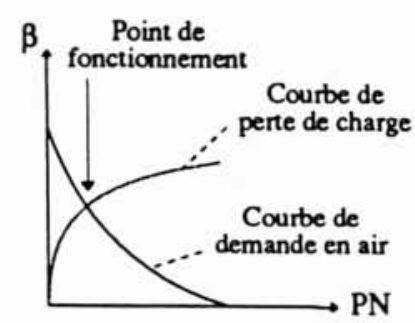

14. Courbe de demande en air d'un aérateur.

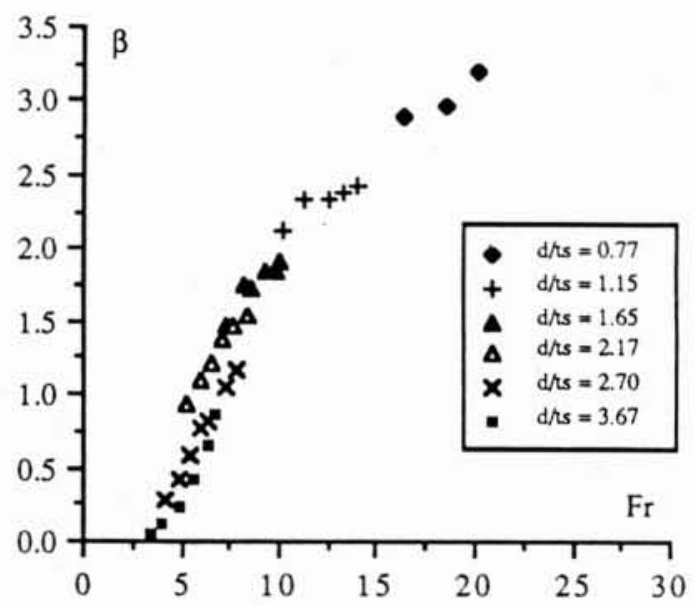

16. Demande en air pour $P_{N}=0$. Aérateur avec un déflecteur $\left(\phi=5.7^{\circ}\right)$. CHANSON (1988).

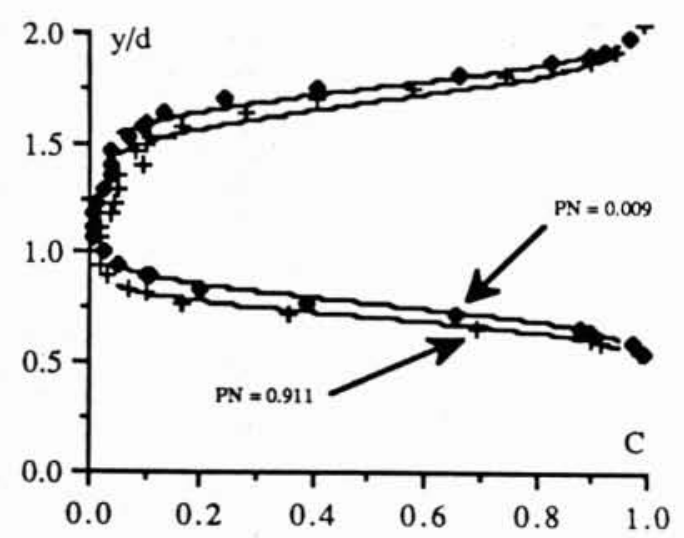

18. Comparaison des mesures de concentrations d'air avec les distributions gaussiennes $\mathrm{Fr}=14,9-d_{0}=34,8 \mathrm{~mm}$. 
Dans le cas particulier d'un aérateur de fond, 1) l'angle d'impact du jet libre ( ig. 13) avec la surface libre (quasi horizontale) des rouleaux $\xi$, 2) l'effet de la proximité du fond de l'évacuateur de crues, et 3) le taux d'aération des interfaces du jet libre doivent être considérés comme paramètres additionnels dans l'étude de l'entrainement d'air par jet plongeant.

Recirculation d'air: Quand le système d'alimentation en air de l'aérateur est obturé $\left(Q_{\text {air }}^{\text {vent }}=0\right)$, ou pour de longs jets libres, l'analyse des mesures de concentration en air (LOW 1986, ChANSON 1988) montre l'existence d'une recirculation d'air dans la cavité. Un tel processus de recirculation avait déjà été observé par ERviNE et AHMED (1982).

Il semble que la recirculation d'air s'effectue par un processus de dé-aération des rouleaux à travers leur surface libre. Les mesures de concentration en air indiquent que les rouleaux sont une mixture di-phasique très aérée $\left(C_{\text {rollcr }}>40 \%\right)$, avec une présence importante de larges bulles d'air $(D=5$ à $10 \mathrm{~mm})$, et ils sont soumis à un fort gradient de pression dû à la pression d'impact du jet (fig. 11).

\subsection{Etude du comportement d'un aérateur de fond}

L'étude de l'entrainement d'air au-dessus d'un aérateur est très étroitement liée à la demande en air de l'aérateur et aux calculs de trajectoire du jet libre. Pour des conditions d'écoulement et une géométrie d'aérateur fixées :

1) l'étude sur modèle de la demande en air d'un aérateur fournit la relation entre le débit d'air de ventilation $Q_{\text {air }}^{\text {vent }}$, et la dépression $\Delta P$ dans la cavité située sous le jet,

2) la connaissance de la courbe de perte de charge du système d'alimentation en air de la cavité (calculée à partir de IDELCHICK [1969]), associée à la courbe de demande en air de l'aérateur $Q_{\text {air }}^{\text {vent }}=f(\Delta P)$, caractérise le point de fonctionnement $\left(Q_{\text {air }}^{\text {vent }}, \Delta P\right)$ de l'aérateur (fig. 14), et

3) les calculs de trajectoires de jet libre (SCHWARTZ et NUIT 1963, TAN 1984, CHANSON 1988) déterminent, pour une dépression $\Delta P,:$ a) la position du point d'impact du jet, b) l'angle d'impact du jet avec la surface de l'évacuateur de crues et c) la géométrie complète de la cavité ventilée.

\section{Etude de l'entraînement d'air}

\subsection{La demande en air}

La demande en air d'un aérateur est définie comme la relation entre le débit d'air fourni par l'alimentation en air, la dépression dans la cavité et les caractéristiques de l'écoulement.

Kobus (1984), Pinto (1984), LAali and Michel (1984) indiquent que les effets de viscosité et de tension superficielle influencent peu la demande en air si : 1) le nombre de Reynolds $\operatorname{Re}=V d / \nu$ est supérieur à $10^{5}$, et 2) le nombre de Weber supérieur à 400 . Dans ces conditions, et pour une configuration géométrique donnée, la relation adimensionnelle de demande en air devient :

$$
\beta^{\mathrm{vent}}=\frac{Q_{\text {air }}^{\mathrm{vent}}}{Q_{\text {cau }}}=f\left(F r=\frac{V}{\sqrt{g d}}, P_{N}=\frac{\Delta P}{\rho_{\text {cau }} g d}\right)
$$

où $V$ est la vitesse moyenne de l'écoulement, et $d$ la profondeur.

Pour différentes profondeurs d'écoulement, les résultats expérimentaux peuvent être présentés comme $1-\beta^{\text {vent }}=f\left(P_{N}\right)$ pour différents nombres de Froude (fig. 15), ou $2-\beta^{\text {vent }}=f(F r)$ pour différents gradients de pression (fig. 16).

La relation entre les trois nombres adimensionnels $\beta^{\text {vent }}, \mathrm{Fr}$ et $P_{N}$ indique qu'une réduction du débit d'air provoque une augmentation de la dépression dans la cavité, et à gradient de pression constant, le débit d'air augmente avec le nombre de Froude. Le graphique 16 montre de plus l'existence d'un changement de mécanisme d'entrainement d'air pour $F r \sim 5$.

Pour des petits nombres de Froude $(F r<5)$, le débit d'air de ventilation naturelle est faible pour toute valeur du gradient de pression. La majeure partie de l'entraînement d'air s'effectue par jet plongeant à l'arrière de la cavité.

\subsection{Etude de la zone d'aération}

LOw (1986) and CHANSON (1988) effectuèrent des mesures de concentrations en air et de vitesses au-dessus et en aval d'un aérateur de fond, et l'auteur développât une méthode permettant de calculer la quantité d'air entraînée dans l'écoulement, dans une section transverse, entre $C=0$ et $C=0,90$. Pour une concentration en air supérieure à $90 \%$, l'écoulement est une projection de gouttes d'eau dans l'air, et les vitesses de chacune des phases (eau et air) ne sont pas égales.

\section{Distribution de concentration en air}

Quand le jet libre quitte le déflecteur, la région d'aération est caractérisé par l'existence d'un noyau central du jet non aéré (fig. 17). Dans cette région (appelée zone d'aération des surfaces libres), la combination de l'équation de conservation de la masse avec l'équation de diffusion devient :

$$
\frac{\mathrm{D}}{\mathrm{D} t} C=\operatorname{div}(D \overrightarrow{\operatorname{grad}} C)-\nabla\left(C \vec{u}_{r}\right)
$$

où $D$ est une valeur moyenne de la diffusivité turbulente, et $u_{r}$ la vitesse limite d'une bulle d'air (équation 6). Les intégrations de l'équation (8) pour les interfaces supérieure et inférieure sont des distributions gaussiennes (ChANSON 1988).

Ceci est en agrément avec les données expérimentales (fig. 18). Il est possible d'obtenir une estimation moyenne de la diffusivité $D$ à l'interface supérieure et inférieure, et les résultats, pour de faibles gradients de pression $(P N<0,01)$, sont présentés dans le tableau 1 (voir page suivante). 


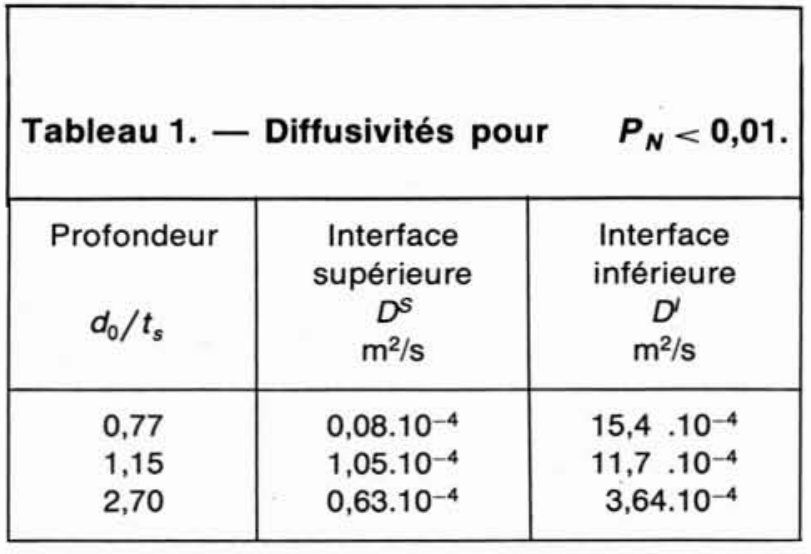

Ces résultats montrent que la diffusivité moyenne à l'interface inférieure $\left(D^{I}\right)$, combinant les effets de la turbulence et du gradient de vitesse, est dix fois plus importante que la diffusivité turbulente à la surface libre supérieure du jet $\left(D^{S}\right)$.

\section{Etude de la quantité d'air entraînée}

Considérons le volume de contrôle $A B C D E F$ (fig. 19), pour lequel les interfaces eau-air $A B$ et $A F$ sont définies pour $C=90 \%$. L'équation de conservation de la masse appliquée à ce volume est :

$$
q_{\text {air }}^{C D}-q_{\text {air }}^{A B}=q_{\text {air }}^{\text {sup }}+q_{\text {air }}^{\text {inf }}+q_{\text {air }}^{\text {plong }}-q_{\text {air }}^{\text {recirc }}
$$

où $q_{\mathrm{air}}^{A B}$ et $q_{\mathrm{air}}^{C D}$ sont les quantités d'air entraînées par le flot à travers les sections $A B$ et $D C, q_{\text {air }}^{\text {sup }}$ est la quantité nette d'air échangée à travers l'interface supérieure $(B C), q_{\text {air }}^{\text {inf }}$ est la quantité nette d'air échangée à travers l'interface inférieure $(A F), q_{\text {air }}^{\text {recirc }}$ est le débit d'air recirculant dans la cavité, et $q_{\text {air }}^{\text {plong }}$ est la quantité d'air entraînée par jet plongeant.

L'équation de conservation de la masse, appliquée à la cavité (volume AFE) est :

$$
\frac{Q_{\mathrm{air}}^{\mathrm{vent}}}{W}+q_{\mathrm{air}}^{\text {recirc }}=q_{\mathrm{air}}^{\mathrm{inf}}+q_{\mathrm{air}}^{\text {plong }}
$$

où $W$ est la largeur du canal. La combinaison des deux précédentes équations devient :

$$
q_{\mathrm{air}}^{C D}-q_{\mathrm{air}}^{A B}=q_{\mathrm{air}}^{\text {sup }}+\frac{Q_{\mathrm{air}}^{\mathrm{vent}}}{W} .
$$

Le terme de gauche caractérise la quantité nette d'air entraînée dans l'écoulement au long du jet libre. L'équation (11) montre que, théoriquement, ce terme peut être à partir de la demande en air $\left(Q_{\text {air }}^{\text {vent }}\right)$ et de l'entraînement net d'air à travers la surface libre supérieure ( $\left.q_{\text {air }}^{\text {sup }}\right)$. Toutefois ce dernier paramètre ne peut pas être mesuré.

A partir des mesures de concentration en air, il est possible d'estimer la quantité d'air entrainée dans l'écoulement :

$$
\beta^{\text {Total }}=\frac{\int_{C=0}^{C=0,9} C V \mathrm{~d} y}{q_{\text {eau }}}
$$

et la quantité d'air entrainé dans la partie supérieure du jet libre :

$$
\beta^{S}=\frac{\int_{C=C_{\min }}^{C=0.9} C V \mathrm{~d} y}{q_{\mathrm{cau}}}
$$

où $C_{\min }$ est la concentration en air minimum dans une section du jet libre. Dans le cas du jet libre, la quantité totale d'air entraînée est définie entre les interfaces $C=90 \%$ :

$$
\beta^{\text {Total }}=\frac{\int_{C=0,9}^{C=0,9} C V \mathrm{~d} y}{q_{\mathrm{eau}}} .
$$

Un exemple est présenté en figure 20 , où $L$ est la distance au long de l'évacuateur de crues depuis la fin du déflecteur, et $d_{0}$ la profondeur initiale.

Les résultats expérimentaux (CHANSON 1988) montrent que l'entraînement d'air à travers l'interface supérieure du jet augmente quand le gradient de pression $P_{N}$ augmente et ceci peut être causé par l'effet du gradient de pression sur la vitesse limite des bulles d'air (équation 6). Ces résultats indiquent en outre que l'entraînement d'air au long de l'interface inférieure est peu affecté par un changement de gradient de pression $P_{N}$.

De ce fait, la quantité totale d'air entraînée $\left(\beta^{\text {total }}\right)$ dans l'écoulement augmente quand le gradient de pression $P_{N}$ augmente, c'est-à-dire quand le débit d'air de ventilation $\beta^{\text {vent }}$ diminue. Mais l'accroissement du gradient de pression augmente la courbure du jet, et donc l'angle d'impact du jet libre avec la surface du coursier et ceci sera discuté plus loin.

\subsection{La zone d'impact}

La zone d'impact est caractérisée par un changement rapide de la pression hydrostatique à la surface du coursier (fig. 11), ainsi que par une rapide redistribution des profils de vitesses et de concentration en air.

Les résultats expérimentaux obtenus sur modèle (fig. 20) montrent que l'écoulement est très fortement dé-aéré et il est suggéré que le fort gradient de pression d'impact du jet en soit responsable.

Les mesures obtenues sur modèle montrent que la quantité d'air entraîné dans l'écoulement à la fin de la zone d'impact, est uniquement fonction de la profondeur initiale (tabl. 2). Il est remarquable que ces résultats soient obtenus indépendamment du débit d'air fourni par l'alimentation en air de l'aérateur $Q_{\text {air }}^{\text {vent }}$.

Pour des conditions d'écoulement similaires, une comparaison des résultats obtenus par Low (1986) et CHANSON (1988) montrent (tabl. 2) que la présence d'un déflecteur affecte peu la quantité d'air entrainé dans l'écoulement en aval de l'aérateur. Avec un défecteur, la quantité d'air entraînée au long du jet libre est plus importante, mais l'angle d'impact du jet avec la surface du coursier de crues augmente aussi et ceci amplifie le gradient de pression d'impact, et donc le processus de dé-aération dans la zone d'impact. 


\begin{tabular}{|c|c|c|c|c|c|}
\hline \multicolumn{6}{|c|}{ Tableau 2.} \\
\hline $\begin{array}{c}\text { Profondeur } \\
\text { initiale } \\
d_{0} / t_{s}\end{array}$ & $\begin{array}{l}\text { Concentration en air à } \\
\text { la fin de la zone d'impact } \\
\qquad C_{m}\end{array}$ & $\begin{array}{c}\text { Profondeur à la fin de } \\
\text { la zone d'impact } \\
\qquad d^{*} / t_{s}\end{array}$ & $\begin{array}{l}\text { Pente } \\
\qquad \alpha\end{array}$ & Conditions & Référence \\
\hline $\begin{array}{l}0,77 \\
1,15 \\
2,70\end{array}$ & $\begin{array}{l}0,32 \\
0,26 \\
0,12\end{array}$ & $\begin{array}{l}0,87 \\
1,10 \\
2,10\end{array}$ & $\begin{array}{l}52,33^{\circ} \\
52,33^{\circ} \\
52,33^{\circ}\end{array}$ & $\begin{array}{l}\text { Sans déflecteur } \\
\text { Sans déflecteur } \\
\text { Sans déflecteur }\end{array}$ & $\begin{array}{l}\text { Chanson (1988b) } \\
\text { Chanson (1988b) } \\
\text { Chanson (1988b) }\end{array}$ \\
\hline 1,67 & 0,29 & 1,15 & $51,30^{\circ}$ & Déflecteur $\phi=5,7^{\circ}$ & Low (1986) \\
\hline
\end{tabular}

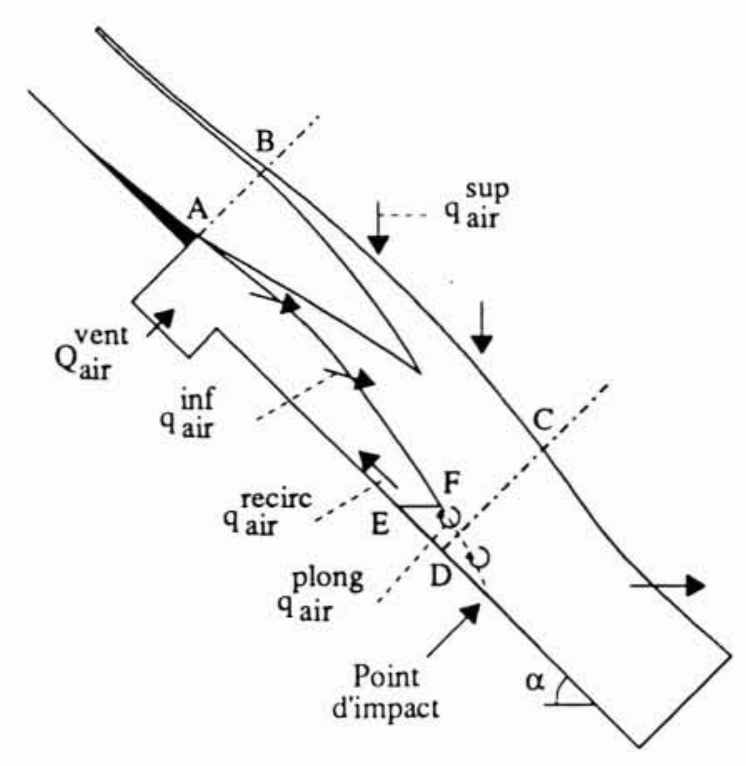

19. Volume de contrôle $A B C E D F$.

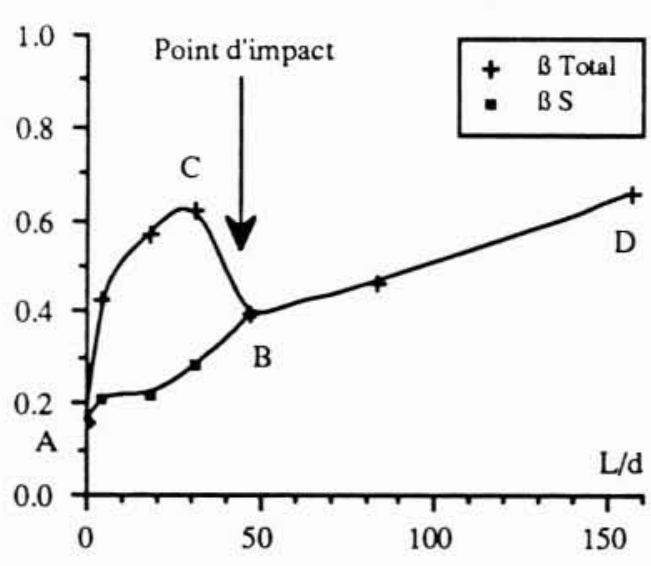

a. $\beta^{\mathrm{vent}}=0,68-P_{N}=0,01$.

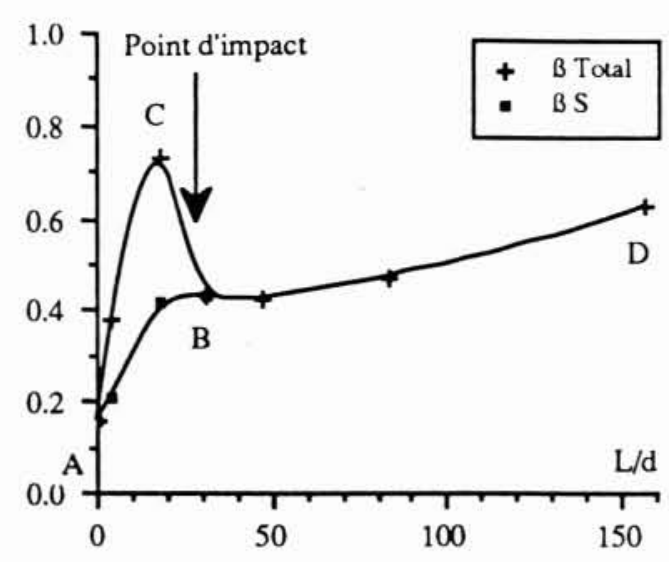

b. $\beta^{\mathrm{vent}}=0,00-P_{n}=0,74$.

20. Quantité d'air entraînée dans l'écoulement pour $d_{0}=$ $22,9 \mathrm{~mm}$ et $\mathrm{Fr}=19,5$. CHANSON (1988). 


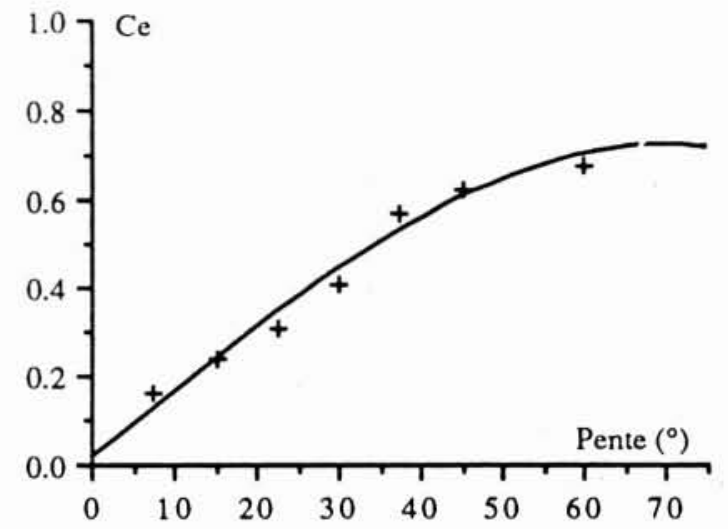

21. $C_{e}$ en fonction de la pente du canal. WOOD (1985).

\section{La région d'écoulement uniforme}

\subsection{Distribution de la concentration en air}

Straub et ANDERSON (1958) ont publié une série complète de mesures de concentration en air, dans un canal à pente variable, pour un écoulement uniforme, sujet à un entraînement d'air naturel à la surface libre. La ré-analyse de ces données par Wood (1985) montre que la concentration en air moyenne dans une section n'est fonction que de la pente du canal, pour un écoulement uniforme (fig. 21 et $t a b l .3$ ). Cette concentration en air moyenne, notée $C_{e}$, est appelée la concentration en air moyenne d'équilibre.

L'analyse de l'équation de conservation de la masse pour la phase air, par WoOD (1984), montre que la distribution de la concentration en air dans la région d'écoulement uniforme s'exprime :

$$
C=\frac{B^{\prime}}{B^{\prime}+e^{-\left(G^{\prime} \cos \alpha y^{\prime 2}\right)}}
$$

où $y^{\prime}$ est la profondeur adimensionnelle $\left(y^{\prime}=Y / Y_{90}\right)$, et $B^{\prime}$ et $G^{\prime}$ sont des constantes fonction de concentration en air moyenne $C_{e}$ (tabl. 3). Les mesures expérimentales de STRAUB et ANDERSON (1958) sont comparées avec l'équation (12) en figure 22.

\subsection{Coefficient de perte de charge}

Il a été suggéré plus haut que : 1) la concentration en air est nulle à la surface de l'évacuateur de crues, et 2) il existe une couche limite de concentration en air. Cette couche limite de concentration en air est plus fine que la couche limite de vitesse. La présence d'air dans la couche limite de vitesse devrait tendre à réduire les contraintes de cisaillement entre les lignes de courant, et donc le coefficient de perte de charge $f$.
Pour un écoulement uniforme, si la distribution de concentration en air est caractérisée par la concentration en air moyenne d'équilibre $C_{e}$, le coefficient de perte de charge est fonction de (WOOD 1984) :

$$
f_{e}=f\left(\frac{d_{e}}{k_{s}}, R e, C_{e}\right)
$$

où $f_{e}$ est le coefficient de perte de charge linéaire pour un écoulement uniforme $\left(f_{e}=\frac{8 g \sin \alpha d_{e}^{3}}{q_{\text {eau }}^{2}}\right), \alpha$ est la pente de l'évacuateur de crues, $d_{c}$ est la profondeur de référence de l'écoulement uniforme, $k_{s}$ est la rugosité uniforme équivalente et $q_{\text {cau }}$ est le débit d'eau par unité de largeur.

$\mathrm{Si}$ les variations de la rugosité sont ignorées, les valeurs de $f_{e}$ calculées à partir des résultats de STRAUB et ANDERSON (1958) indiquent que le coefficient de perte de charge diminue quand la concentration en air moyenne augmente (fig. 23). Le coefficient de perte de charge décroît à partir d'une concentration en air moyenne $C_{e}$ supérieure à $20 \%$, ce qui correspond à une concentration en air, près de la surface du coursier, supérieure à $5 \%$ (fig. 10).

Il n'y a pas, à présent, d'informations sur les variations de $f_{e}$ avec la rugosité. Toutefois, si l'équation (13) est réécrite comme :

$$
f_{e}=f_{1}\left(\frac{d_{e}}{k_{s}}, R e\right) f_{2}\left(C_{e}\right)
$$

et si on utilise la condition limite pour $C=0: f_{e}=f$, WoOD (1984) montre que :

$$
\frac{f_{e}}{f}=f_{2}\left(C_{e}\right) \text {. }
$$

\subsection{Profil de vitesses}

CAIN (1978) effectua des mesures de vitesses dans des écoulements à aération naturelle, sur l'évacuateur de crues du barrage d'Aviemore (Nouvelle-Zélande). Ces résultats sont remarquables en ce que la distribution des vitesses peut être écrite, comme suggérée par WOOD (1984) :

$$
\frac{V}{V_{90}}=\left(\frac{y}{Y_{90}}\right)^{\frac{1}{6}} .
$$

Les points expérimentaux, dont dépend cette équation, sont portés sur la figure 24 . Il faut noter que ces points ont été obtenus pour des concentrations en air moyennes variant entre 0 et $50 \%$. Il apparaît alors que le profil de vitesses est indépendant de la concentration en air. Ce résultat est obtenu dans une région d'écoulement lentement varié, et, comme il est indépendant de la concentration en air moyenne, il est raisonnable de supposer que ce résultat s'applique aussi à la région d'équilibre (WOOD 1985). 
Tableau 3. Distribution adimensionnelle de concentration en air. STRAUB et ANDERSON (1958).

\begin{tabular}{|c|c|c|c|c|}
\hline & Pente & $C_{e}$ & $G^{\prime *} \cos \alpha$ & $B^{\prime}$ \\
\hline$\bullet$ & $7,5^{\circ}$ & 0,161 & 7,99952 & 0,00302 \\
$\diamond$ & $15,0^{\circ}$ & 0,241 & 5,74469 & 0,02880 \\
$\mathbf{\square}$ & $22,5^{\circ}$ & 0,310 & 4,83428 & 0,07157 \\
$\square$ & $30,0^{\circ}$ & 0,410 & 3,82506 & 0,19635 \\
$\Delta$ & $37,5^{\circ}$ & 0,569 & 2,67484 & 0,62026 \\
$\Delta$ & $45,0^{\circ}$ & 0,622 & 2,40096 & 0,81568 \\
$\times$ & $60,0^{\circ}$ & 0,680 & 1,89421 & 1,35393 \\
+ & $75,0^{\circ}$ & 0,721 & 1,57440 & 1,86418 \\
\hline
\end{tabular}

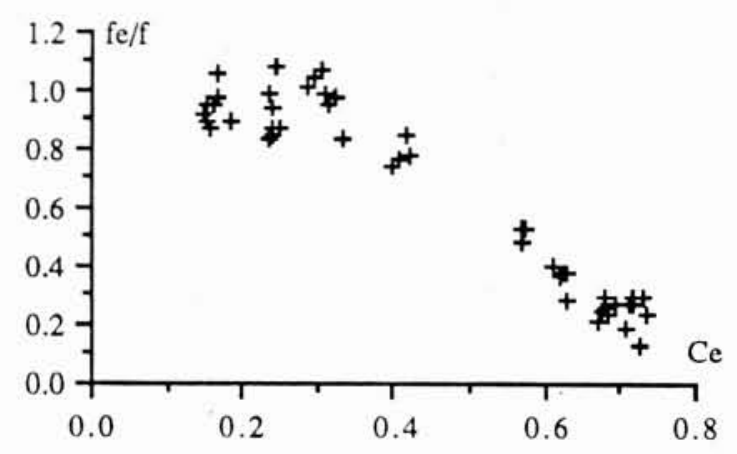

23. $f_{e} / f$ en fonction de $C_{e}$ à partir des mesures de STRAUB et ANDERSON (1958) WOOD (1984).

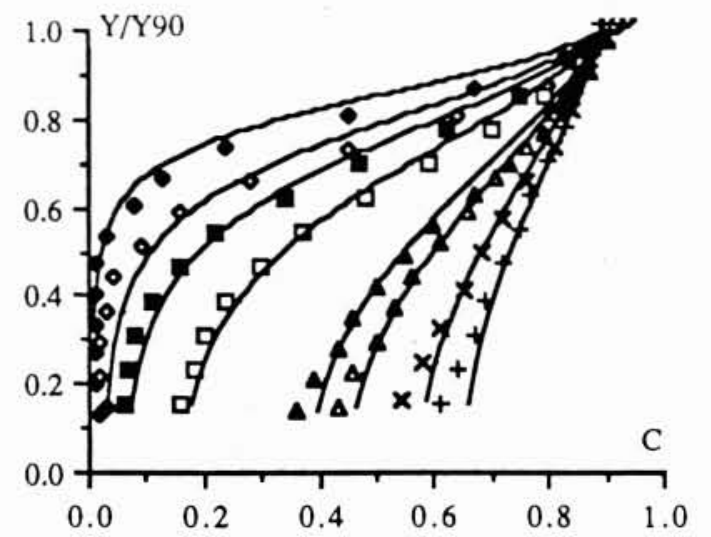

22. Profils de concentration en air. STRAUB et ANDERSON $\left(q_{\mathrm{cau}}=0,136 \mathrm{~m}^{2} / \mathrm{s}\right)$.

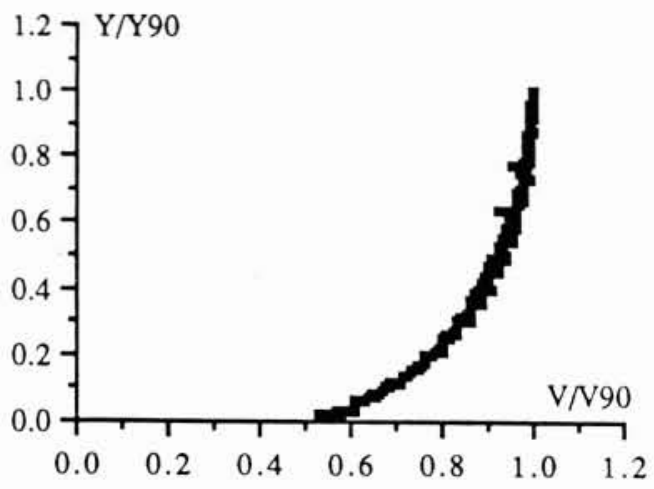

24. Profil de vitesses à Aviemore CAIN (1978).

canal $\alpha$ donnée, la figure 15 fournit une estimation de la concentration en air moyenne d'équilibre $C_{e}$. Si la valeur du coefficient de perte de charge $f$, pour un écoulement sans aération naturelle, est disponible, la figure 23 donne le coefficient de perte de charge $f_{e}$, pour l'écoulement aéré. On en déduit la profondeur de référence de l'écoulement uniforme $d_{e}$ :

$$
d_{e}=\left[\frac{q_{\mathrm{cau}}^{2} f_{e}}{8 g \sin \alpha}\right]^{1 / 3} .
$$

La vitesse moyenne de l'écoulement $V_{m}$ et la profondeur de référence $Y_{90}$ sont déduites des équations [2] et [3]. Les distributions de vitesses et de concentrations en air sont obtenues à partir des équations (12), (15) et (16).

Ainsi que l'a détaillé Wood (1985), pour une pente de 


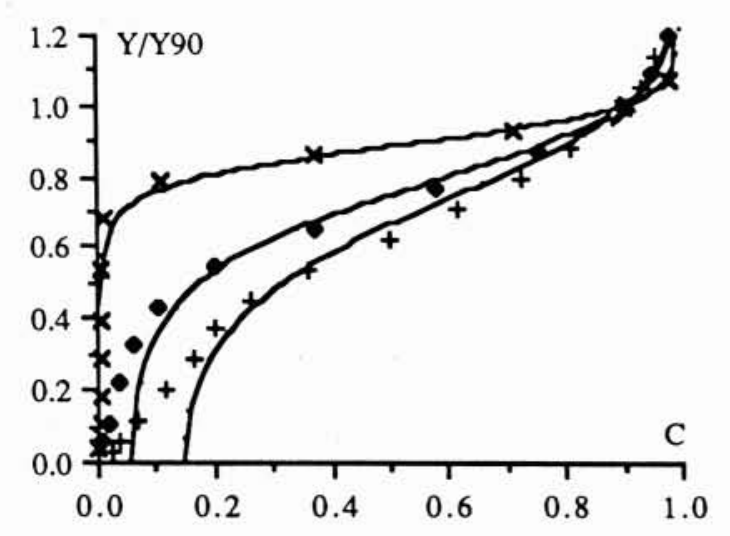

\begin{tabular}{|c|c|c|c|}
\hline Ref. & $\begin{array}{c}q_{\text {eau }} \\
\mathrm{m}^{3} / \mathrm{s} / \mathrm{m}\end{array}$ & $C_{m}$ & $\begin{array}{c}d \\
\mathrm{~m}\end{array}$ \\
\hline$+860-1$ & 0,2118 & 0,3952 & 0,0212 \\
$\times 873-1$ & 0,2731 & 0,2957 & 0,0258 \\
$\times 880-1$ & 0,4291 & 0,1203 & 0,0491 \\
\hline
\end{tabular}

25. Mesures de concentration en air en aval d'un aérateur. CHANSON (1988).

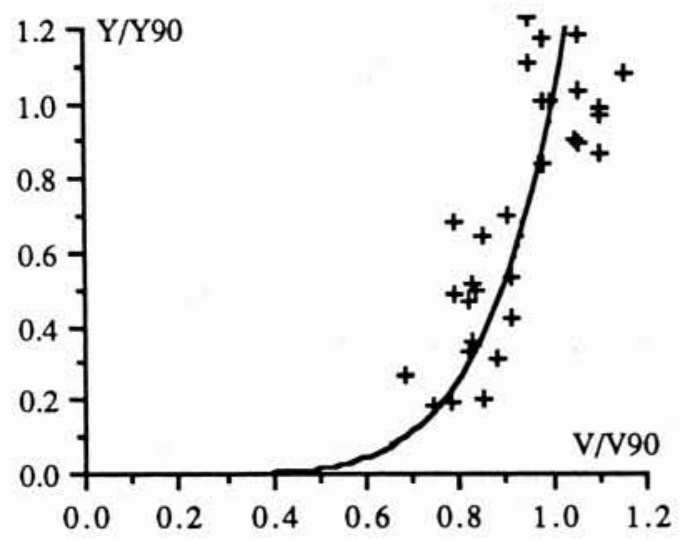

26. Profils de vitesses en aval d'un aérateur. CHANSON (1988).

\section{Ecoulement en aval d'un aérateur}

\subsection{Introduction}

Wood (1985) a montré que, si l'on suppose : $\{H 1\}$ un taux d'entrainement d'air faible, $\{H 2\}$ une distribution de pression quasi hydro-statique, et $\{H 3\}$ des variations graduelles (ou lentes) de la distribution des vitesses, l'équation de conservation de la masse pour la phase air et l'équation d'énergie fournissent deux équations permettant de calculer la concentration en air moyenne, et la profondeur de référence $d$ (équation [1]) en tout point d'un écoulement graduellement varié, en aval du point d'inception de l'entraînement d'air.

L'auteur (CHANSON 1988b) a montré que, si les hypothèses $H 1, H 2$ et $H 3$ sont respectées, ces équations, développées par WoOD (1985), peuvent s'appliquer à un écoulement graduellement varié en aval d'un aérateur. Dans une première partie, les principales équations vont être présentées, et seront ensuite appliquées aux écoulements en aval d'un aérateur. Ces résultats permettront de définir une méthode pour la disposition et location des aérateurs sur un évacuateur de crues.

\subsection{Fonction d'entraînement}

Dans la région d'écoulement graduellement varié, en aval d'un aérateur, les mesures de concentration en air (ChAnson 1988a) montrent un faible taux d'entraînement d'air, et ceci suggère que, pour une concentration en air moyenne $C_{m}$, la distribution de concentration en air suit une distribution proche de celle d'un écoulement uniforme. Cette hypothèse est confirmée par la comparaison des résultats expérimentaux avec l'équation (12) (fig. 25).

Les mesures de vitesses obtenues par CAIN (1978) ont été faites dans une région d'écoulement graduellement varié, en aval du point d'inception, et il semble raisonnable de supposer que l'équation (15) puisse s'appliquer à tout écoulement graduellement varié. L'auteur (CHANSON 1988b) observa, sur modèle, des distributions de vitesses similaires, en aval d'un aérateur (fig. 26). Ces mesures expérimentales sont comparées avec l'équation (15), et les erreurs sont plus importantes que pour CAIN à cause des limitations de l'instrumentation.

Avec la même méthode, que celle développée par WoOD (1985), en aval du point d'inception, l'équation de conservation de la masse pour la phase air est :

$$
\frac{\mathrm{d}}{\mathrm{d} x} q_{\mathrm{air}}=V_{e}(x)-C_{m}(x) u_{r} \cos \alpha
$$

où $u_{r}$ est la vitesse limite d'ascension des bulles d'air dans la région d'écoulement graduellement varié, $V_{e}$ est la vitesse d'entraînement d'air (WooD 1985), $C_{m}$ la concentration en air moyenne locale, et $\alpha$ la pente du coursier.

En utilisant la limite de l'équation (17) pour la région d'écoulement uniforme :

$$
0=\bar{V}_{e}-C_{e} u_{r} \cos \alpha
$$


l'équation de conservation de la masse pour la phase air est ré-écrite :

$\frac{\mathrm{d}}{\mathrm{d} x} q_{\mathrm{air}}=\left(V_{e}(x)-\bar{V}_{e}\right)+\left(C_{e}-C_{m}(x)\right) u_{r} \cos \alpha$.

WoOD (1985) propose de négliger le premier terme, et l'équation (18) devient :

$$
\frac{\mathrm{d}}{\mathrm{d} x} q_{\mathrm{air}}=\left(C_{e}-C_{m}(x)\right) u_{r} \cos \alpha .
$$

La forme de cette équation indique que, pour une concentration en air moyenne $C_{m}$ inférieure à la concentration en air d'équilibre $C_{e}$, l'écoulement est aéré. Par contre, lorsque la concentration en air moyenne est supérieure à la concentration en air d'équilibre, l'écoulement est dé-aéré.

Chanson (1988) a montré que la quantité d'air entraîné dans l'écoulement peut être ré-écrite :

$$
q_{\mathrm{air}}=\frac{C_{m}}{1-C_{m}} q_{\mathrm{cau}} .
$$

Avec ces approximations, l'équation de conservation de la masse pour la phase air devient :

$$
\frac{\mathrm{d}}{\mathrm{d} x} C_{m}=\frac{u_{r} \cos \alpha}{q_{\mathrm{cau}}}\left(C_{\mathrm{e}}-C_{m}\right)\left(1-C_{m}\right)^{2} .
$$

L'intégration de l'équation (13) conduit à :

$$
\frac{1}{\left(1-C_{e}\right)^{2}} \mathrm{LN}\left[\frac{1-C_{m}}{C_{e}-C_{m}}\right]-\frac{1}{\left(1-C_{e}\right)\left(1-C_{m}\right)}=k x K_{0}
$$

où $K_{0}$ est une constante d'intégration déterminée par $C_{m}=C^{*}$ à $x=0$ :

$$
\begin{aligned}
K_{0}= & \frac{1}{\left(1-C_{e}\right)} \times \\
& \quad \times\left(\frac{1}{\left(1-C_{e}\right)} \mathrm{LN}\left[\frac{1-C_{m}}{C_{e}-C_{m}}\right]-\frac{1}{\left(1-C^{*}\right)}\right)
\end{aligned}
$$

et

$$
k=u_{r} \cos \alpha / q_{\text {cau }} \text {. }
$$

Les mesures expérimentales obtenues par CAIN (1978) en aval du point d'inception et par l'auteur (1988b) en aval d'un aérateur ont été utilisées pour contrôler la validité des équations ci-dessus (CHANSON 1988).

Pour deux valeurs de débits, les résultats de CAIN vérifient l'équation (20) avec une corrélation supérieure à 0,95 , et ils impliquent des valeurs de la vitesse limite d'ascension d'une bulle d'air $u_{r}=40 \mathrm{~cm} / \mathrm{s}$. Ces valeurs, obtenues en aval du point d'inception, pour un écoulement à aération naturelle, sont proches des valeurs dans un liquide au repos, publiées par COMOLET (1979).

Les résultats expérimentaux obtenus sur modèle en aval d'un aérateur (CHANSON 1988), impliquent des valeurs plus faibles de $u_{r}$ entre 1 à $16 \mathrm{~cm} / \mathrm{s}$. Ceci peut être en partie expliquer par le niveau élevé de la turbulence en aval d'un aérateur: HaINDL (1969) indique que le rapport $u_{r}^{t} / u_{r}$ peut atteindre 0,25 , où $u_{r}^{t}$ est la vitesse limite des bulles d'air dans un écoulement turbulent, et $u_{r}$ la vitesse limite pour un liquide au repos.
Le niveau élevé de turbulence en aval de la zone d'impact devrait décroître logiquement, et loin en aval de l'aérateur, et dans la région d'écoulement uniforme, ce niveau pourrait être similaire à celui observé dans un écoulement à surface libre avec aération naturelle. De ce fait, l'auteur (CHANSON 1988) suggère que la vitesse limite d'une bulle d'air, dans un écoulement graduellement varié en aval d'un aérateur, devrait tendre lentement, d'une valeur initiale en écoulement turbulent (ex. : $u_{r}^{t} \# 0.25 u_{r}$ ), vers une valeur comparable à celles observées pour un écoulement à surface libre (ex. : $u_{r}=40 \mathrm{~cm} / \mathrm{s}$ à Aviemore).

Les résultats obtenus sur modèle en aval d'un aérateur doivent être utilisés avec beaucoup de circonspection, car : 1) des paramètres additionnels, comme la distribution de la turbulence, sont encore inconnus, et 2) des effets d'échelle de réduction peuvent affecter ces résultats.

Finalement, si on considère la vitesse limite d'ascension des bulles d'air comme une constante locale variant lentement, l'équation (19) peut être ré-écrite en terme adimensionnel :

$$
\frac{\mathrm{d}}{\mathrm{d} x^{\prime}} C_{m}=\frac{u_{r} \cos \alpha}{q_{\text {cau }}}\left(C_{e}-C_{m}\right)\left(1-C_{m}\right)^{2} \mathrm{~d}^{*}
$$

où $d^{*}$ est la profondeur de référence pour $x=0$, $x^{\prime}=x / d^{*}, u_{r}$ et $q_{\text {cau }}$ sont des constantes, et $C_{e}$ est une fonction de $\alpha$ seulement. L'équation (21) est remarquable, car elle permet de calculer la concentration en air moyenne $C_{m}$ en fonction de la distance $x$ au long du coursier, indépendamment de la vitesse, de la rugosité et de la profondeur.

\subsection{Equation d'énergie}

WooD (1985) développa l'équation d'énergie pour une ligne de courant à une profondeur $y$ au-dessus du coursier :

$$
\begin{aligned}
& E(y)=\rho(y) g(z+y \cos \alpha)+ \\
& \quad+\int_{y}^{\infty} \rho(h) g \cos \alpha \mathrm{d} h+\rho(y) \frac{[V(y)]^{2}}{2} .
\end{aligned}
$$

En supposant que : 1) le champ de pression est quasi hydrostatique, et 2 ) le profil de vitesses vérifie :

$$
q_{\mathrm{eau}} d=\int_{0}^{\gamma_{90}}(1-C) y V(y) \mathrm{d} y
$$

l'énergie spécifique moyenne devient :

$$
S E=z+d \cos \alpha+E \frac{d}{2} F r^{2}
$$

où $y^{\prime}=\frac{y}{Y_{90}}, V^{\prime}=\frac{V}{V_{90}}, F r=\frac{V_{m}}{\sqrt{g d^{*}}}$ et le paramètre $E$ est défini comme (WoOd [1985]) :

$$
E=\left(1-C_{m}\right)^{2} \frac{\int_{0}^{1}(1-C) V^{\prime 2} \mathrm{~d} y^{\prime}}{\left[\int_{0}^{1}(1-C) V^{\prime} \mathrm{d} y^{\prime}\right]^{3}} .
$$




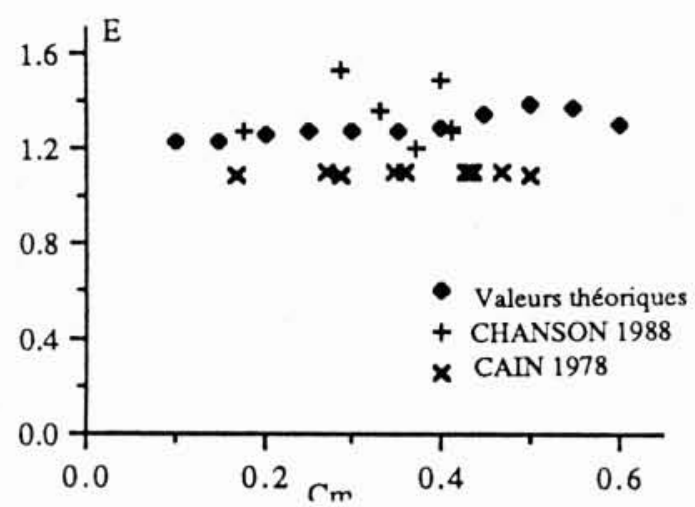

27. Paramètre $E$ en fonction de la concentration en air moyenne.

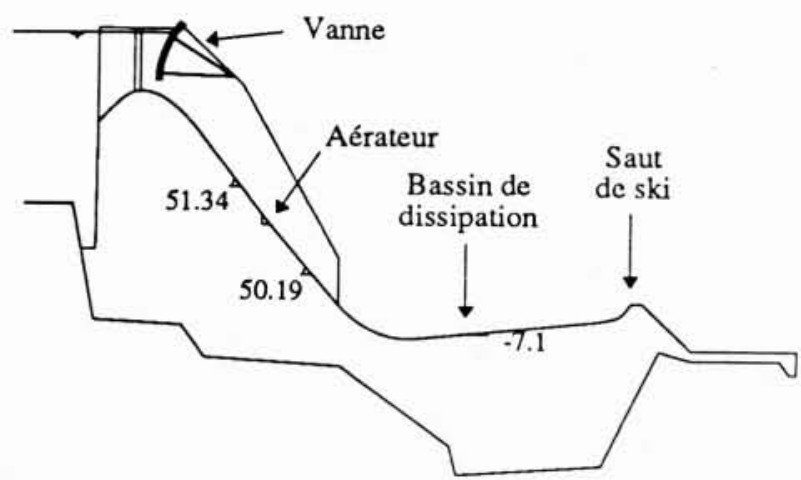

29. Evacuateur de crues du barrage de Clyde (NouvelleZélande).

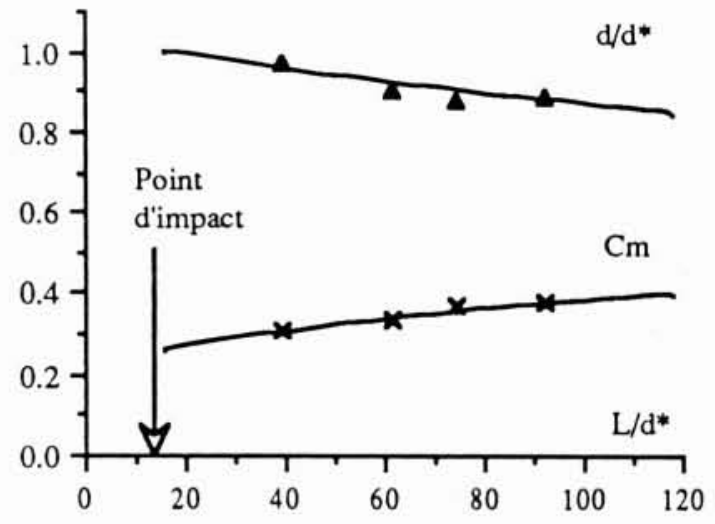

28. Entraînement d'air en aval d'un aérateur.

$q_{\mathrm{cau}}=0,40 \mathrm{~m}^{3} / \mathrm{s} / \mathrm{m}-d_{0} / t_{\mathrm{s}}=1,15 u_{r}=16 \mathrm{~cm} / \mathrm{s}-d^{*}=$ $0,039 \mathrm{~m}=-C^{*}=0,26$.

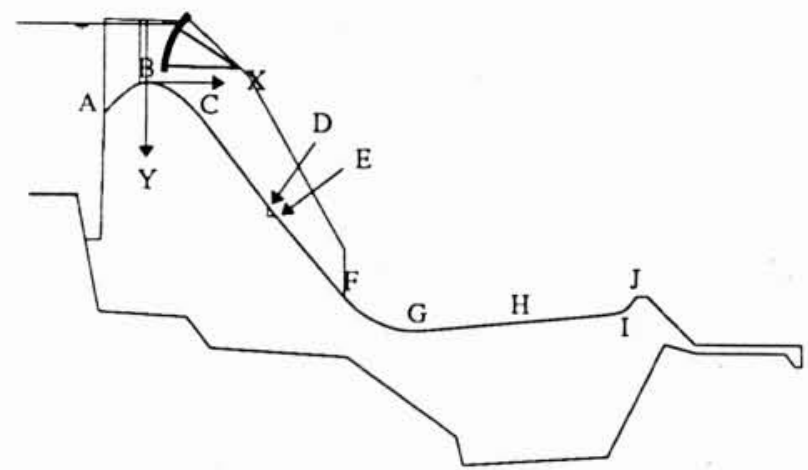

30. Points caractéristiques de l'évacuateur de crues du barrage de Clyde (Nouvelle Zélande).

Tableau 4. Evacuateur de crues du barrage de Clyde. Points caractéristiques

\begin{tabular}{|c|c|c|c|c|c|c|}
\hline Point & $\begin{array}{l}\text { Elévation } \\
\mathrm{m}\end{array}$ & $\begin{array}{l}Y \\
m\end{array}$ & $\begin{array}{l}x \\
m\end{array}$ & $\alpha$ & $\begin{array}{l}S \\
m\end{array}$ & Remarque \\
\hline$A$ & & 6,90 & $-8,50$ & $-45,0^{\circ}$ & $-11,2$ & \\
\hline$B$ & 182,00 & 0,00 & 0,00 & $0,00^{\circ}$ & 0,00 & Origine. \\
\hline$C$ & 172,16 & 9,84 & 14,00 & $51,34^{\circ}$ & 17,53 & \\
\hline$D$ & & 26,15 & 27,06 & $51,34^{\circ}$ & 38,42 & Fin du déflecteur de l'aérateur. \\
\hline$E$ & 153,00 & 29,00 & 28,75 & $50,19^{\circ}$ & 41,74 & Fin de la rainure de l'aérateur. \\
\hline$F$ & 135,20 & 46,80 & 43,59 & $50,19^{\circ}$ & 64,91 & $\begin{array}{l}\text { Fin du coursier. Arrondi } \\
\text { circulaire : } R=20 \mathrm{~m} \text {. }\end{array}$ \\
\hline$G$ & & 54,11 & 62,09 & $-7,125^{\circ}$ & 85,65 & Début du bassin de dissipation. \\
\hline$H$ & & 51,71 & 81,28 & $-7,125^{\circ}$ & 105,00 & \\
\hline 1 & & 49,01 & 102,85 & $-7,125^{\circ}$ & 126,73 & $\begin{array}{l}\text { Fin du bassin de dissipation. } \\
\text { Arrondi circulaire : } R=1,5 \mathrm{~m} \text {. }\end{array}$ \\
\hline$J$ & 137,00 & 45,00 & 111,07 & $-68,20^{\circ}$ & 135,93 & Fin du saut de ski. \\
\hline
\end{tabular}


Pour un écoulement graduellement varié, le profil des vitesses est estimé à partir de l'équation (15), et le paramètre $E$ devient :

$$
E=\left(1-C_{m}\right)^{2} \frac{\int_{0}^{1}(1-C) y^{2^{\frac{2}{6}}} \mathrm{~d} y^{\prime}}{\left[\int_{0}^{1}(1-C) y^{\prime^{\frac{1}{6}}} \mathrm{~d} y^{\prime}\right]^{3}}
$$

où $C$ est calculée à partir de l'équation (12). $E$ est alors uniquement fonction de la concentration en air moyenne $C_{m}$. Les valeurs théoriques (équation 25) de $E$ sont reportées en figure 27 , et comparées avec les résultats calculés à partir des mesures de CAIN (1978), à Aviemore, en aval du point d'inception, et de CHANSON (1988), en aval d'un aérateur, sur un modèle 1/15ème.

Pour un canal avec des variations lentes de pente et de largeur, et en combinant l'équation de conservation de la quantité de mouvement :

$$
\frac{\mathrm{d}}{\mathrm{d} x} S E=S_{f}
$$

avec l'équation (23), nous obtenons :

$$
\frac{\mathrm{d}}{\mathrm{d} x} d=\frac{\sin \alpha\left(1+d \frac{\mathrm{d} \alpha}{\mathrm{d} x}\right)-S_{f}+\frac{E d}{W} F r^{2} \frac{\mathrm{d} W}{\mathrm{~d} x}}{\cos \alpha-E F r^{2}}
$$

Les variations lentes des vitesses et des concentrations en air suggèrent que le coefficient de perte de charge en écoulement uniforme peut être utilisé comme une valeur locale (WOOD 1985), et le coefficient $S$, devient :

$$
S_{f}=\frac{q_{\mathrm{cau}} f}{8 g d^{3}} f_{2}\left(C_{m}\right)
$$

où la fonction $f_{2}$ est obtenue à partir de la figure 23 . L'équation (26) peut être ré-écrite en terme adimensionnel :

$$
\frac{\mathrm{d}}{\mathrm{d} x^{\prime}} d^{\prime}=\frac{\sin \alpha\left(1+d^{\prime} \frac{\mathrm{d} \alpha}{\mathrm{d} x^{\prime}}\right)-S_{f}+\frac{E}{W^{\prime}} \frac{F r^{2}}{d^{\prime 2}} \frac{\mathrm{d} W^{\prime}}{\mathrm{d} x^{\prime}}}{\cos \alpha-E \frac{F r^{2}}{d^{\prime 3}}}
$$

où $d=d^{*}$ pour $x=0, x^{\prime}=\frac{x}{d^{*}}, d^{\prime}=\frac{d}{d^{*}}, W^{\prime}=\frac{W}{d^{*}}$, et $F r^{*}=\frac{q_{\mathrm{cau}}}{\sqrt{g d^{3}}}$.

L'équation (27) est une équation différentielle, où les valeurs locales de $S_{f}$ et $E$ sont fonction de la concentration en air moyenne.

\subsection{Application}

\subsubsection{Présentation}

Les équations (21) et (27) fournissent un système de deux équations différentielles où les deux variables sont la profondeur de référence d et la concentration en air moyenne $C_{m}$. La connaissance de d et $C_{m}$ en tout point de l'évacuateur de crues permet de calculer les distributions de concentration en air (équation 12) et le profil des vitesses (équations 15 et 16) au long de l'évacuateur de crues.

Dans le cas particulier d'un coursier à largeur constante, les paramètres sont obtenus à partir d'un système simple d'équations :

$$
\begin{aligned}
\frac{\mathrm{d}}{\mathrm{d} x^{\prime}} C_{m} & =\frac{u_{r} \cos \alpha}{q_{\mathrm{cau}}}\left(C_{e}-C_{m}\right)\left(1-C_{m}\right)^{2} d^{*} \\
\frac{\mathrm{d}}{\mathrm{d} x^{\prime}} d^{\prime} & =\frac{\sin \alpha\left(1+d^{\prime} \frac{\mathrm{d} \alpha}{\mathrm{d} x^{\prime}}\right)-S_{f}}{\cos \alpha-E \frac{F r^{2}}{d^{\prime 3}}}
\end{aligned}
$$

qui peut être résolu numériquement (CHANSON 1988). Ces équations ont été utilisées pour reproduire l'entraînement d'air en aval d'un aérateur, sur un modèle $1 / 15$ ème et pour un débit particulier (fig. 28). Les conditions initiales proviennent du tableau 1, et l'origine du graphe est prise à la fin du déflecteur.

\subsubsection{Application de la méthode proposée à l'évacuateur de crues du barrage de Clyde}

L'évacuateur de crues du barrage de Clyde (NouvelleZélande) comprend un coursier de $70 \mathrm{~m}$ de long (pente : $\alpha=51.34^{\circ}$ et $50.19^{\circ}$ ), suivi d'un bassin de dissipation (longueur: $50 \mathrm{~m}$ ), avec une pente négative (pente : $\alpha=-7,1^{\circ}$ ). et finissant par un saut de ski. Le coursier est équipé d'un aérateur, situé $39 \mathrm{~m}$ en dessous du niveau du réservoir, et $28 \mathrm{~m}$ au-dessus du bassin de dissipation (fig. 29). La position des points caractéristiques (fig. 30), dans l'axe de l'évacuateur de crues, est reportée sur le tableau 4 , où $S$ désigne la distance curviligne depuis le point $B$.

Les équations (28) et (29) déterminent l'entraînement d'air sur le coursier et le bassin de dissipation, et il est possible de comparer l'entraînement d'air pour un coursier sans ou avec aérateur. Il faut noter que l'équation (29) a été obtenue pour un champ de pression quasi hydrostatique. La transition $F-G$ entre le coursier et le bassin de dissipation présente un arrondi circulaire (rayon: $R=20 \mathrm{~m}$ ). HENDERSON $(1966)^{1}$ montre que l'augmentation de pression due à un changement de direction est faible pour $d / R>10$ (pour $R=20 \mathrm{~m}$, $d<2 \mathrm{~m}$ ). De ce fait, l'hypothèse d'une distribution hydrostatique de pression est raisonnable pour $d<2 \mathrm{~m}$.

Pour un débit $q_{\text {cau }}=21 \mathrm{~m}^{3} / \mathrm{s} / \mathrm{m}$, la rugosité uniforme équivalente $k_{\mathrm{s}}=3 \mathrm{~mm}$ correspond à un coefficient de perte de charge $f=0,025$.

La seule valeur de vitesse limite d'ascension d'une bulle d'air, obtenue sur prototype, est le résultat $(u,=40 \mathrm{~cm} / \mathrm{s})$ calculé à partir des mesures de CAIN (1978) sur le barrage d'Aviemore. Cette valeur est utilisée pour les calculs d'écoulement graduellement varié en aval du point d'inception (coursier sans aérateur). L'extrapolation des résultats de la vitesse limite d'ascension d'une bulle d'air $u_{r}$, obtenus sur modèle, est sujette à des effets d'échelle de réduction, et de ce fait la valeur de $u_{r}$ en aval d'un aérateur sur prototype est prise égale à celle mesurée à Aviemore : $u_{r}=40 \mathrm{~cm} / \mathrm{s}$. 
Tableau 5. Résultats pour $q_{\text {eau }}=21 \mathrm{~m}^{3} / \mathrm{s} / \mathrm{m}$ et $u_{r}=40 \mathrm{~cm} / \mathrm{s}$.

\begin{tabular}{|c|c|c|c|c|c|c|c|c|c|}
\hline Point & $\begin{array}{l}S \\
\mathrm{~m}\end{array}$ & $\alpha$ & $\begin{array}{l}d \\
\mathrm{~m}\end{array}$ & $C_{m}$ & $\begin{array}{l}d \\
\mathrm{~m}\end{array}$ & $C_{m}$ & $\begin{array}{l}d \\
\mathrm{~m}\end{array}$ & $C_{m}$ & Remarque \\
\hline & & & \multicolumn{2}{|c|}{ Sans aérateur } & \multicolumn{2}{|c|}{ Aérateur en $\mathrm{D}$} & \multicolumn{2}{|c|}{ Aérateur en C } & \\
\hline & 30,13 & $51,34^{\circ}$ & & & & & 1,43 & 0,26 & Début de l'écoulement \\
\hline & 56,92 & $50,19^{\circ}$ & & & 0,720 & 0,26 & & & $\begin{array}{l}\text { Début de l'écoulement } \\
\text { graduellement varié. }\end{array}$ \\
\hline$F$ & 64,91 & $50,19^{\circ}$ & 0,675 & 0,00 & 0,701 & 0,28 & 0,878 & 0,33 & Fin du coursier. \\
\hline G & 85.65 & $-7,125^{\circ}$ & 0,687 & 0,07 & 0,710 & 0,28 & 0,851 & 0,32 & Début du bassin de dissipation. \\
\hline$H$ & 105,00 & $-7,125^{\circ}$ & 0,750 & 0,05 & 0,774 & 0,23 & 0,928 & 0,27 & \\
\hline 1 & 126,73 & $-7,125^{\circ}$ & 0,826 & 0,04 & 0,853 & 0,17 & 1,026 & 0,21 & Fin du bassin de dissipation. \\
\hline$J$ & 135,93 & $-68,20^{\circ}$ & 0,903 & 0,03 & 0,938 & 0,16 & 1,157 & 0,19 & Fin du saut de ski. \\
\hline
\end{tabular}

31. Profil de concentration en air au point $H . q_{\mathrm{cau}}=$ $21 \mathrm{~m}^{3} / \mathrm{s} / \mathrm{m}-u_{r}=40 \mathrm{~cm} / \mathrm{s}$.

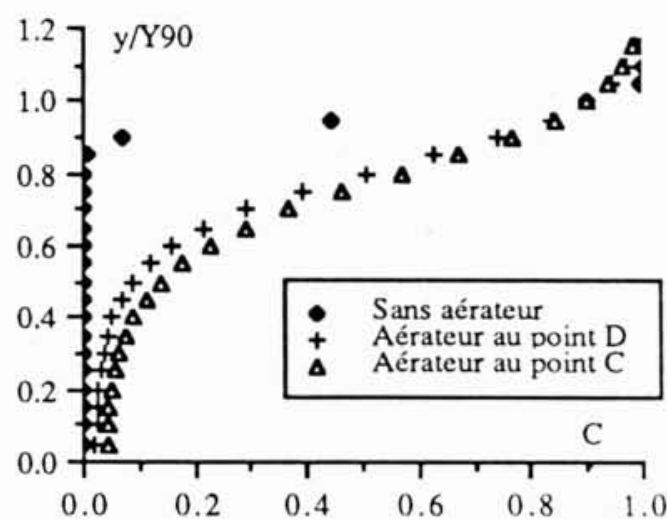

Pour ce débit $q_{\text {cau }}=21 \mathrm{~m}^{3} / \mathrm{s} / \mathrm{m}$, et cette rugosité uniforme équivalente $k_{s}=3 \mathrm{~mm}$, le point d'inception d'entraînement d'air, pour un coursier sans aérateur, est déterminé par Keller et RASTOGi (1977). Ces calculs montrent que l'entraînement d'air commence approximativement au point $F$ et la profondeur au point d'inception est $d_{l}=0,675 \mathrm{~m}$.

Pour le débit considéré, la profondeur de référence dans la zone d'approche de l'aérateur est $d_{0}=0,75 \mathrm{~m}$ au poind $D$. Le point d'impact du jet libre, calculé à partir de Chanson (1988), est situé $13,5 \mathrm{~m}$ en aval du point $D$. En supposant que la région d'écoulement graduellement varié commence $18,5 \mathrm{~m}$ en aval du point $D$, les conditions initiales $C^{*}$ et $d^{*}$ sont déduites des résultats sur modèle (tabl. 2) : $C^{*}=0,26$ et $d^{*}=0,72 \mathrm{~m}$.

Les résultats sont regroupés dans le tableau 5. Au point $H$ situé au milieu du bassin de dissipation, les distributions de concentration en air pour le coursier sans aérateur, ou avec aérateur (au poind $D$ ) sont représentées sur le graphique 31 .

Les résultats indiquent que, sans aérateur, l'érosion par cavitation se produirait sur le coursier et le bassin de dissipation, car les concentrations en air près du fond sont inférieures à $6-8 \%$. Pour ce débit particulier, la présence de l'aérateur (au poind $D$ ) devrait protéger le coursier et la plupart du bassin de dissipation, mais des dommages peuvent se produire à la fin du bassin de dissipation (entre les points $H$ et $J$ ).

Il faut remarquer que ces résultats dépendent critiquement des hypothèses sur les valeurs de la vitesse limite d'une bulle d'air $u_{r}$, du coefficient de perte de charge $f$, de la concentration en air initiale $C^{*}$, et de la profondeur initiale $d^{*}$.

\subsection{Disposition des aérateurs}

\subsubsection{Présentation}

Pour prévenir l'érosion par cavitation du coursier, il est important de connaître la redistribution des concentrations en air, en aval d'un aérateur, et dans les couches d'eau proches de la surface du coursier. Dans la région d'écoulement graduellement varié, en aval de la zone d'impact, les profils de concentration en air tendent vers 
un profil d'équilibre, fonction de la pente locale, et ceci peut être déterminé à partir des équations (21) et (27).

L'érosion par cavitation peut se développer à la surface du coursier, si la concentration en air près de cette surface est inférieure à $5-10 \%$, ce qui correspond (fig. 22) à une concentration en air moyenne $C_{m}$ inférieure à $30 \%$.

Sur un coursier raide $\left(\alpha>20^{\circ}\right)$, les concentrations en air tendront vers un profil d'équilibre tel que $C_{e}>30 \%$ (tabl. 2). Si la concentration en air moyenne au début de l'écoulement graduellement varié est suffisamment importante $\left(C_{m}>25-30 \%\right)$, toute la longueur du coursier en aval du premier aérateur sera protégée, et il ne sera pas nécessaire d'installer un second aérateur tant que $\alpha>20^{\circ}$. Si la pente du coursier devient inférieur à $20^{\circ}$, ou pour un coursier à pente douce $\left(\alpha<20^{\circ}\right)$, l'écoulement en aval de l'aérateur sera dé-aéré, et un (ou plusieurs) aérateur(s) devra être installé au point où la concentration en air $C_{m}$ deviendra inférieur à $30 \%$.

\subsubsection{Application}

Quand l'aération naturelle de l'écoulement, à travers la surface libre, est insuffisante, ou si l'érosion par cavitation peut se développer en amont du point d'inception de l'entraînement d'air, il est alors nécessaire d'installer un aérateur.

Un aérateur de fond introduit artificiellement, et sur une distance relativement courte, une quantité importante d'air dans l'écoulement (CHANSON 1988). La quantité d'air entraîné dans l'écoulement est fonction du dimensionnement de l'aérateur (tabl. 2).

Dans le cas d'un coursier à pente faible, et comme le flot sera dé-aéré en aval de l'aérateur, le premier aérateur devra être disposé légèrement en amont de la zone critique, sujette à cavitation.

Pour un coursier à pente raide, il conviendra de placer l'aérateur le plus en amont possible de façon à combiner l'aération artificielle de l'écoulement par l'aérateur avec l'aération naturelle de l'écoulement en aval de l'aérateur.

Dans le cas du barrage de Clyde, nous avons étudié le positionnement virtuel de l'aérateur au point $C$ ( fig. 30). Pour le même débit $q_{\text {cau }}=21 \mathrm{~m}^{3} / \mathrm{s} / \mathrm{m}$ précédemment étudié, la profondeur en amont de l'aérateur serait $d_{0}=1,5 \mathrm{~m}$, et le point d'impact du jet libre, calculé à partir de CHANSON (1988), serait situé $8,4 \mathrm{~m}$ en aval du point $C$. En supposant que la région d'écoulement graduellement varié commencerait $12,6 \mathrm{~m}$ en aval du point $C$, les conditions initiales $C^{*}$ et $d^{*}$ ont été choisies : $C^{*}=0,26$ et $d^{*}=0,72 \mathrm{~m}$.

Les résultats (tabl.5) indiquent une aération plus importante de l'écoulement. La distribution de concentration en air au point $H$ (milieu du bassin de dissipation) est portée sur la figure 31. Il est possible de comparer ces résultats avec les résultats obtenus pour le coursier sans aérateur, et avec aérateur au point $D$ : la concentration en air, à $20 \mathrm{~mm}$ du fond, calculé à partir de l'équation (5), serait : $C=0 \%$ sans aérateur, $C=2 \%$ pour l'aérateur au point $D$, et $C=4 \%$ avec un aérateur au point $C$.

\section{Conclusion}

Pour un aérateur de fond, les phénomènes d'entrainement d'air sont caractérisés par un entraînement d'air interfaciale le long des surfaces libres supérieure et inférieure du jet libre, un entraînement d'air additionnelle par jet plongeant et un processus de recirculation d'air dans la cavité située sous l'interface inférieure du jet.

Les résultats expérimentaux sur modèle montrent que l'entraînement d'air à travers la surface libre supérieure du jet est fortement dépendant du gradient de pression entre les interfaces, alors que le rapide changement des contraintes de cisaillement semble un paramètre dominant à la surface libre inférieure. Les phénomènes de recirculation d'air et d'entraînement d'air par jet plongeant dans le cas d'un aérateur de fond, sont encore mal connus, et des études complémentaires sont nécessaires.

Dans la zone d'impact, l'écoulement est soumis à des redistributions importantes des profils de vitesses et de concentration en air, et il est sujet à une forte déaération. Les mesures obtenues sur modèle indiquent que les conditions d'écoulement en aval de cette zone sont pratiquement indépendamment du point de fonctionnement $\left(Q_{\text {air }}^{\text {vent }}, \Delta P\right)$ de l'aérateur. De plus amples mesurements dans cette région sont toutefois nécessaires sur prototype, pour confirmer ces résultats.

Une complète analogie entre l'écoulement graduellement varié en aval d'un point d'inception, et l'écoulement graduellement varié en aval d'un aérateur a été développée. La ré-interprétation d'une analyse développée par WOOD (1985), appliquée à l'écoulement en aval d'un aérateur, fournit un système de deux équations différentielles, pouvant être résolues numériquement.

Ces équations reproduisent l'entrainement d'air en aval d'un aérateur, et permettent d'estimer en tout point les distributions de vitesses et de concentration en air, ainsi que le taux d'aération (ou de dé-aération) de l'écoulement. A partir de ces résultats, il est possible d'estimer la position optimale du ou des aérateur(s).

Il faut remarquer que l'estimation de la vitesse limite d'ascension d'une bulle d'air, dans un écoulement graduellement varié en aval d'un aérateur, nécessite des études supplémentaires sur prototype.

\section{Remerciements}

L'auteur tient à remercier le département de Génie civil de I'Université de Canterbury (Nouvelle-Zélande), l'University Grant Committee (Nouvelle-Zélande) et le Ministère du Travail et Développement (NouvelleZélande), qui ont financé ce projet, ainsi que le Professeur I. R. WoOD, qui supervisa cette étude. 


\section{Notations}

C : Concentration en air définie comme le ratio du volume d'air sur le volume total.

$C_{d} \quad$ : Coefficient de trainée d'une bulle de gaz.

$C_{e} \quad$ : Concentration en air moyenne dans la région d'écoulement uniforme.

$C_{m} \quad$ : Concentration en air moyenne dans une section.

$C^{*} \quad$ : Concentration en air moyenne au début de la région d'écoulement.

d : Profondeur de référence mesurée perpendiculairement à la surface libre $(\mathrm{m})$.

$d_{b} \quad$ : Diamètre d'une bulle de gaz $(\mathrm{m})$.

$d_{e} \quad$ : Profondeur de référence d'un écoulement uniforme $(\mathrm{m})$.

$d_{0} \quad$ : Profondeur de référence initiale $(\mathrm{m})$.

$d^{*} \quad$ : Profondeur de référence au début de la région d'écoulement graduellement varié $(\mathrm{m})$.

$D^{\prime} \quad$ : Diffusivité moyenne à l'interface inférieure $\left(\mathrm{m}^{2} / \mathrm{s}\right)$.

$D^{S} \quad$ : Diffusivité turbulente à l'interface supérieure $\left(\mathrm{m}^{2} / \mathrm{s}\right)$.

$f \quad$ : Coefficient de perte de charge linéaire.

$f_{e} \quad$ : coefficient de perte de charge pour un écoulement uniforme.

Fr : Nombre de Froude.

$g$ : Constante de gravité $\left(\mathrm{m} / \mathrm{s}^{2}\right)$.

$k_{\text {s }} \quad$ : rugosité uniforme équivalente $(\mathrm{m})$.

$L$ : Distance au long de l'évacuateur de crues depuis la fin du déflecteur $(\mathrm{m})$.

$L_{\mathrm{jct}} \quad$ : Longueur du jet libre $(\mathrm{m})$.

$P_{\text {atm }}$ : Pression atmosphérique $(\mathrm{Pa})$.

$P_{N} \quad$ : Gradient adimensionnel de pression.

$q_{\mathrm{Nair}} \quad$ : Quantité d'air entraîné dans l'écoulement $\left(\mathrm{m}^{3} / \mathrm{s} / \mathrm{m}\right)$.

$q_{\text {air }}^{\text {inf }}:$ Quantité nette d'air échangée à travers l'interface inférieure $\left(\mathrm{m}^{2} / \mathrm{s} / \mathrm{m}\right)$.

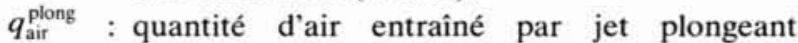
$\left(\mathrm{m}^{3} / \mathrm{s} / \mathrm{m}\right)$.

$q_{\text {air }}^{\text {recire }}$ : débit d'air recirculant dans la cavité $\left(\mathrm{m}^{3} / \mathrm{s} / \mathrm{m}\right)$.

$q_{\text {air }}^{\text {sup }}$ : Quantité nette d'air échangée à travers l'interface supérieure $\left(\mathrm{m}^{3} / \mathrm{s} / \mathrm{m}\right)$.

$q_{\text {air }}^{S} \quad$ : Quantité d'air entraîné dans la partie supérieure de l'écoulement $\left(\mathrm{m}^{3} / \mathrm{s} / \mathrm{m}\right)$.

$Q_{\text {air }}^{\text {vent }}$ : Débit en air de ventilation $\left(\mathrm{m}^{3} / \mathrm{s}\right)$.

$q_{\text {cau }}$ : Débit en eau par unité de largeur $\left(\mathrm{m}^{3} / \mathrm{s} / \mathrm{m}\right)$.

$Q_{\text {cau }}:$ Débit total en eau $\left(\mathrm{m}^{3} / \mathrm{s}\right)$.

Re : Nombre de Reynolds.

$S$ : Coordonnée curviligne (m).

$t_{s} \quad$ : Hauteur de la marche $(\mathrm{m})$.

$u_{r} \quad$ : Vitesse limite d'ascension d'une bulle d'air $(\mathrm{m} / \mathrm{s})$.

$v \quad$ : Volume $\left(\mathrm{m}^{3}\right)$

$v_{\text {air }} \quad$ : Volume en air $\left(\mathrm{m}^{3}\right)$.

$v_{\text {cau }} \quad$ : Volume en eau $\left(\mathrm{m}^{3}\right)$.

$V \quad:$ Vitesse $(\mathrm{m} / \mathrm{s})$

$V_{\text {air }}:$ Vitesse de la phase air $(\mathrm{m} / \mathrm{s})$.
$V_{\text {cau }}:$ Vitesse de la phase eau $(\mathrm{m} / \mathrm{s})$.

$V_{m}$ : Vitesse moyenne de l'écoulement $(\mathrm{m} / \mathrm{s})$.

$W \quad$ : Largeur du canal (m).

We : Nombre de Weber.

$x \quad$ : Coordonnée curviligne (m).

$x^{\prime} \quad$ : Coordonnée curviligne adimensionnelle.

$\alpha \quad$ : Pente de l'évacuateur de crues.

$\beta^{\text {vent }}$ : Débit adimensionnel d'air fourni par l'alimentation en air.

$\Delta P \quad:$ Différence de pression entre la pression atmosphérique et la pression dans la cavité située sous l'interface inférieure du jet $(\mathrm{Pa})$.

$\phi \quad$ : Angle du déflecteur.

$v \quad$ : Viscosité cinématique $\left(\mathrm{m}^{2} / \mathrm{s}\right)$.

$\rho_{\text {cau }}:$ Masse volumique de l'eau $\left(\mathrm{kg} / \mathrm{m}^{3}\right)$.

$\rho_{\text {air }} \quad$ : Masse volumique de l'air $\left(\mathrm{kg} / \mathrm{m}^{3}\right)$.

$\sigma \quad$ : Tension interfaciale eau-air $(\mathrm{N} / \mathrm{m})$.

\section{Références}

P. CAIN, 1978: Measurements within Self-Acrated Flow on a Large Spillway, Ph. D. Thesis, Ref. 78-18, University of Canterbury, Christchurch, New Zealand, 1978.

J. A. Casteleyn, P. A. Kolman et P. Van Groen, 1977 : Air Entrainment in Siphons-Scale Model Tests and an Extrapolation, Delft Hydraulic Laboratories, Hollande, Report n* 1987, 1977.

H. Chanson, 1988 : Study of Air Entrainment and Aeration Devices on Spillway Model, Research Report 8-88, University of Canterbury, New Zealand, October 1988.

R. COMOLET, 1979: Sur le Mouvement d'une bulle d'air dans un liquide, La Houille Blanche, $\mathrm{n}^{*} 1,1979$, pp. 31-42.

D. A. Ervine et A. A. Ahmed, 1982: A Scaling Relationship for a Two-Dimensional Vertical Drop Shaft, Int. Conf. on the Hyd. Modelling in Civ. Eng. Struct., BHRA, Coventry, England, paper E1, 1982.

K. HAINDL, 1969: Zone Lengths of Air Emulsion in Water Downstream of the Ring Jump in Pipes, 13th Cong. IAHR, August 1969, VI 2, pp. 9-19, Kyoto, Japon.

F. M. HENDERSON, 1966: Open Channel Flow, McMillan Co, New York, USA, 1966.

L. E. IDElchick, 1969 : Mémento des Pertes de Charge, Eyrolles, Paris, France, 169.

R. J. Keller et A. K. Rastogi, 1977: Design Chart for Predicting Critical Point on Spillways. Jl of Hyd. Div., ASCE, HY12, Proc. Paper 13426, December 1977 , pp. 1417-1429.

H. KoвUS, 1984 : Local Air Entrainment and Detrainment, Symp. on Scale Effects in Mod. Hyd. Struct., IAHR, Esslingen, West Germany, 1984.

A. R. LAALI et J. M. MiCHEL, 1984: Air Entrainment in Ventilated Cavities: Case of the Fully Developed "Half-Cavity", Trans. of ASME, Jl of Fluids Eng. September 1984, VI 106, pp. 327-335. 


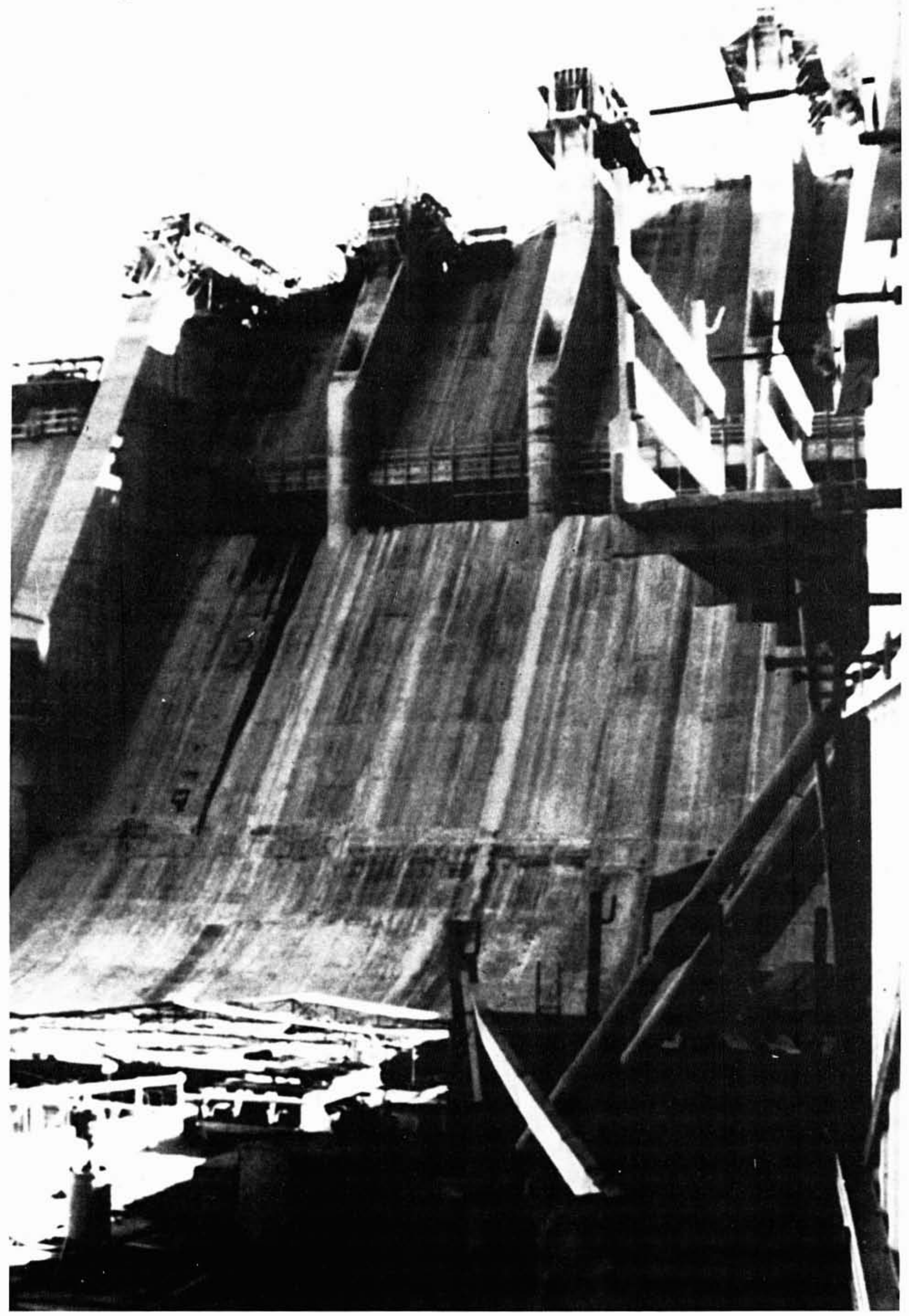

Evacuateur de crues au barrage de Clyde (Nouvelle-Zélande).

Photo prise par l'auteur lors de la construction.

On note la présence de l'aćrateur au milieu du coursier. 
H. S. Low, 1986: Model Studies of Clyde Dam Spillway Aerators, Master Report, Ref. 86-6, University of Canterbury, New Zealand 1986.

A. J. Peterka, 1953 : The effect of Entrained Air on Cavitation Pitting, Joint meeting paper, IAHR/ASCE, Minneapolis, USA, August 1953.

N. L. de S. Pinto, 1984 : Model Evaluation of Aerators in Shooting Flow. Symp. on Scale Effects in Mod. Hyd. Struct., IAHR, Esslingen, West Germany, 1984.

S. O. Russell et G. J. Sheehan, 1974 : Effect of Entrained Air on Cavitation Damage Canadian, $J l$ of Civ. Eng., Vol. 1, 1974.

H. I. Schwartz et L. P. Nutt, 1963 : Projected Nappes subject to Transverse Pressure, Jl of Hyd. Div., Proc. ASCE, July 1963 , pp. $97-104$.

L. G. Straub et A. G. Anderson, 1958 : Experiments on SelfAerated Flow in Open Channel, $\mathrm{Jl}$ of Hydr. Div., ASCE, VI. 84, HY7, 1958.

T. P. TAN, 1984: Model Studies of Aerators on Spillways, Master Report, Ref. 84-6, University of Canterbury, New Zealand, 1984.
P. Volkart et A. Chervet, 1983: Air Slots for Flow Aeration. Mitteilungen der Versuchsanstalt für Wasserbau, Hydrologie und Glaziologie, n 66, Zürich, Suisse, 1983.

P. Volkart et P. Rutschmann, 1984: Air Entrainment Devices. Mittellungen der Versuchsanstalt für Wasserbau, Hydrologie und Glaziologie, n` 72, Zürich, Suisse, 1984.

I. R. Wood, 1984 : Air Entrainment in High Speed Flows. Symp. on Scale Effects in Mod. Hyd. Struct., IAHR, Esslingen, West Germany, 1984.

I. R. WoOD, 1985: Air Water Flows, 21st Congress IAHR, Aug. 1985, Melbourne, Australia.

Adresse de l'auteur:

Monsieur Hubert Chanson,

143, rue de la Pompe, 75116 Paris, France.

Tél. : (33 1) 472722 32. Télécopie: (33 1) 47559943

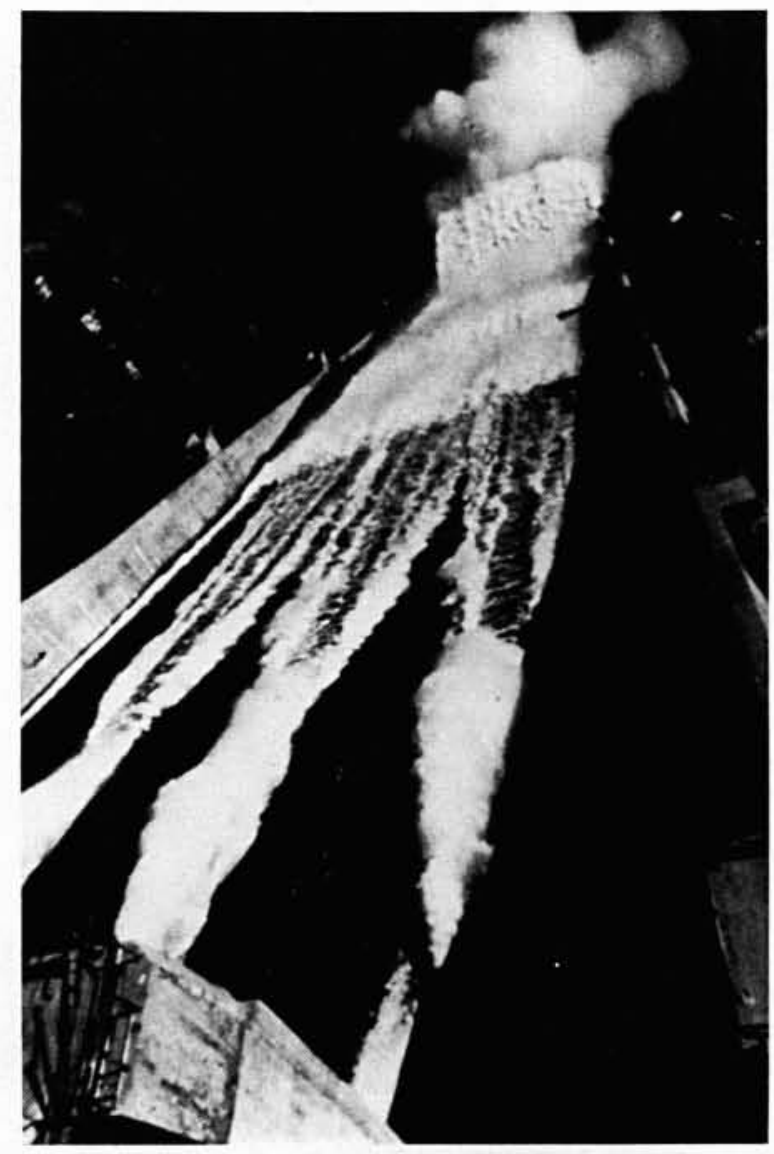

Evacuateur de crues du barrage de Foz de Areia (Brésil).

On note la présence de 3 aérateurs le long du coursier.

Photo aimablement fournic par le professeur I. R. Wood, Université de Canterbury (Nouvelle-Zélande). 